\title{
Crystal Structure of AgSCN Coordination Polymers for Sensing of Iron (III) lons, Nitrobenzene and Photocatalytic Activity Under Ultrasonic Irradiation
}

Safaa Etaiw ( $\sim$ Safaaetaiw@hotmail.com )

Tanta University https://orcid.org/0000-0001-6040-198X

Mohamed M. Dawood

Tanta Higher Institute of Engineering and Technology (THIET)

Hassan Marie

Tanta University

\section{Research Article}

Keywords: Nanosized AgSCN, Crystal structure, Luminescent sensor, Photocatalytic degradation; Acid Blue 92, Eosin-Y, Nitrobenzene.

Posted Date: March 2nd, 2021

DOI: https://doi.org/10.21203/rs.3.rs-253211/v1

License: (c) (i) This work is licensed under a Creative Commons Attribution 4.0 International License. Read Full License 


\title{
Crystal structure of AgSCN coordination polymers for sensing of iron (III) ions, Nitrobenzene and photocatalytic activity under ultrasonic irradiation
}

\author{
Safaa El-din H. Etaiw ${ }^{1}$, Mohamed M. Dawood ${ }^{2}$, Hassan Marie ${ }^{1}$ \\ ${ }^{1}$ Chemistry Department, Faculty of Science, Tanta University, Tanta, Egypt. \\ ${ }^{2}$ Tanta Higher Institute of Engineering and Technology (THIET), Tanta, Egypt.
}

\begin{abstract}
Single crystals of AgSCN supramolecular coordination polymer, SCP1 and the nanosized particles of $\mathrm{AgSCN}, 2$, are synthesized by self-assembly and ultrasonic radiation methods, respectively. The structure comprises of the simple AgSCN molecule fabricated by a tetrahedral Ag atom and one thiocyanate group. The SCN ligand connects four Ag atoms via $\mu 3-\mathrm{S}$ and one nitrogen atom. Two silver atoms and two sulfur atoms form rhombic mini-cycle $\left(\mathrm{Ag}_{2} \mathrm{~S}_{2}\right)$ motif exhibiting argentophilic interaction. The structure of SCP1 extends threedimensionally via coordinate bonds creating different fused polygons. The chemical structure and morphology of nanosized $\mathbf{2}$ are characterized by powder $\mathrm{X}$-ray diffraction and transmission electron microscopy (TEM) and spectroscopic methods. The heterogeneous catalytic and photo-catalytic activity of nanosized $\mathbf{2}$ exhibit very efficient catalytic activity towards degradation of Acid Blue-92 and Eosin-Y dyes within very short times under UVlight or ultrasonic radiation. The good limit of detection and the high quenching efficiency of SCP1 towards selective quantitative determination of nitrobenzene (NB) and $\mathrm{Fe}^{3+}$ ions support its behavior as an efficient luminescent sensor.
\end{abstract}

Keywords: Nanosized AgSCN, Crystal structure, Luminescent sensor, Photocatalytic degradation; Acid Blue 92, Eosin-Y, Nitrobenzene.

*Corresponding author: Prof. Dr. Safaa El-din H. Etaiw

safaaetaiw@ hotmail.com and safaaetaiw@science.tanta.edu.eg

\section{Introduction}


A significant attention was paid in recent years for supramolecular coordination polymers (SCPs) and consequently metal organic frameworks (MOFs) as polymorphic and multipurpose materials [1]. Such materials are assembled using different techniques giving variable and limitless structures with high surface area, tunable optical properties, perpetual porosity and high adsorption affinity $[2,3]$. SCPs showed a great efficiency as heterogeneous catalysts [4], chemical sensors [5, 6], nanofluid additives [7], as well as their effective role in drug delivery [8], storage and separation [1,9]. The high porous nature and surface area enhance the catalytic ability of SCPs. As many chemical processes, the heterogeneous catalysis is one of the main solutions of our developed society [10]. Due to the high efficiency, low cost, and lack of secondary environmental pollution, the photocatalysis has attracted high interest. The discharge of industrial dye polluted wastewater effluents into aquatic sources causes severe problems to human health and impact the aquatic environment. In order to minimize the harmful effects of toxic dyes on humans and the environment, SCPs were used as heterogeneous catalysts for degrade such dyes [5]. Pollution of surface and ground water with heavy metal ions as Fe(III) ions can cause damage and disease to humans or other organisms [11]. Sensitive and selective detection of these ions are of biological, industrial and environmental interest [12]. SCPs are also considered as desirable chemical sensor materials for the detection of small molecular species such as ions in water system [13]. The short response time and high selectivity are the main advantages of luminescent SCPs as chemical sensors $[14,15]$. Silver (I) based SCPs have attracted great concern due to their aesthetic architectures and promising applications where, $\operatorname{Ag}(\mathrm{I})$ has a tendency to form Ag...Ag interaction, which has greatly enriched Ag-SCPs motifs [16]. On the other hand, the thiocyanate compounds were known as good semiconductors because they easily adsorb 
many organic and inorganic substances that this property is essential for photocatalysts and dye-sensitized photovoltaic systems [17]. Here, the synthesis and characterization of single crystals (AgSCN) SCP1 and the nanosized particles 2, will be disused respectively, by selfassembly and sonochemical methods as well as their applications in photodegradation of toxic organic dyes such as Acid Blue 92 (AB-92), scheme 1 and Eosin-Y (EO-Y), scheme 2, as a model pollutants. Additionally, the luminescence behavior of SCP1 is investigated to detect its ability as sensor for selective determination of nitrobenzene and metal ions.

\section{Experimental}

\subsection{Materials and measurements}

All utilized organic solvents and reagents in this study were purchased from Sigma-Aldrich with excessive purity available and were used as received. The analytical and physical measurements for the structural characterization were similar to those used in our previous works $[18,19]$.

\subsection{Synthesis of silver thiocyanate single crystals; ${ }^{3}{ }_{\infty}[\mathrm{AgSCN}], \mathrm{SCP} 1$}

At ambient conditions, three solutions of $\mathrm{AgNO}_{3}$ [1.0 mmol in $15 \mathrm{~mL} \mathrm{H} \mathrm{H}_{2} \mathrm{O}$, KSCN [0.2 mmol in $10 \mathrm{~mL} \mathrm{H} \mathrm{H}_{2} \mathrm{O}$ ], and ethyl iso-nicotinate (EIN) ligand [0.4 mmol in $20 \mathrm{~mL}$ of $\mathrm{CH}_{3} \mathrm{CN}$ and $\mathrm{H}_{2} \mathrm{O}$ (1:1)] were mixed, stirred for 30 min and kept enclosed for two weeks to obtain EIN free colorless prismatic single crystals of silver thiocyanate [AgSCN], SCP1 by self-assembly method. The crystals were filtered off, washed with $\mathrm{CH}_{3} \mathrm{CN} / \mathrm{H}_{2} \mathrm{O}$ (1:1) solvents and then were dried. IR spectrum of SCP1 as $\mathrm{KBr}$ pellet exhibits bands at 3476br, 3097w, 1732s, 1620s, 1588s, 1555s, 1451s $1387 \mathrm{~m}, 1267 \mathrm{~m}, 751 \mathrm{~s}, 647 \mathrm{~m}, 546 \mathrm{~m}, 521 \mathrm{w}, 492 \mathrm{w} \mathrm{cm}{ }^{-1}$, Fig. S1.

\subsection{Synthesis of nanosized 2 silver thiocyanate}


At the same amounts and conditions of SCP1 preparation and through ultrasonic bath, the mixture of the ternary adducts was subjected to ultrasonic radiation for $60 \mathrm{~min}$ at 30 ${ }^{\circ} \mathrm{C}$ with a distinct power of $70 \mathrm{~W}$ to synthesize nanosized particles $\mathbf{2}$. The resultant precipitate was centrifuged, washed with $20 \mathrm{~mL} \mathrm{H}_{2} \mathrm{O}$ and then was dried where about $68 \%$ of nanosized 2 was obtained. The elemental analysis data of AgSCN nanosized 2 (M.W. $=156.95 \mathrm{~g} / \mathrm{mol}$ ) are Anal. Calculated: C, 7.65\%; N, 8.92\%.; S, 20.42\%; Ag, 68.72\%, Found: C, 7.71\%; N, 8.852\%.; S, 20.65\%; Ag, 68.64\%. IR spectrum of SCP1 as $\mathrm{KBr}$ pellet exhibits bands at 3478br, 3098w, 1733s,1622s, 1589s, 1557s, 1452s 1387 m, 1268 m, 752s, 648 m, 546 m, 521w, 493w cm $\mathrm{c}^{-1}$, Fig. S1.

\subsection{Photocatalytic activity measurements of the nanosized 2}

The photocatalytic activity of the nanosized 2 for degradation of both Acid Blue-92 (AB-92) dye $(\lambda=560 \mathrm{~nm})$ and Eosin-Y (EO-Y) dye $(\lambda=514 \mathrm{~nm})$, Schemes S1 and S2, were tested under ambient conditions (AC), UV-light radiation using a $300 \mathrm{~W}$ high pressure mercury lamp and ultrasonic radiation through ultrasonic bath $70 \mathrm{~W}$. Typically, the photocatalytic experiments were carried out by mixing $25 \mathrm{mg}$ of $\mathrm{AgSCN}$ nanosized 2 as a heterogeneous catalyst with $50 \mathrm{~mL}$ aqueous dye solution of AB-92 $\left(5 \times 10^{-5} \mathrm{M}\right)$ or EO-Y $(2.5 \mathrm{x}$ $\left.\left.10^{-4} \mathrm{M}\right)\right]$ in presence of $(0.1 \mathrm{M}) \mathrm{H}_{2} \mathrm{O}_{2}$. The solution was then stirred magnetically in dark chamber for one hour till attains equilibrium between desorption and adsorption. During each process and at definite time, about $5 \mathrm{~mL}$ of solution was continuously taken, filtered off and was analyzing using UV-visible spectrometer. The same procedures were used in control experiments without using any catalyst. Also, the photocatalytic activity of $\mathbf{2}$ in presence of ammonium oxalate (AO), benzoquinone (BQ), and isopropanol (IPA) were investigated under UV-light irradaiton. The catalytic degradation efficiency $(D \%)$ was calculated using Eq. 1, 


$$
D \%=\frac{C}{C_{0}}=\left(\frac{A_{0}-A_{t}}{A_{0}}\right) \times 100
$$

Where $\mathrm{A}_{\mathrm{o}}$ and At are the absorbance of $\mathrm{AB}-92$ or EO-Y dyes at zero time and at time $\mathrm{t}$ (min).

\subsection{The luminescence and sensing properties of SCP1}

A certain amount $(5 \mathrm{mg})$ of SCP1 as fine powder was suspended in $10 \mathrm{~mL}$ of some organic solvents which include DMSO, $\mathrm{MeOH}_{2} \mathrm{CHCl}_{3}, \mathrm{CH}_{2} \mathrm{Cl}_{2}$, THF, EtOH, IPA, $\mathrm{CH}_{3} \mathrm{CN}, \mathrm{DMF}$, $\mathrm{CCl}_{4}$, acetone and nitrobenzene (NB). Then the fluorescence spectra of the suspensions formed by ultrasonic irradiation were measured. Also, the same procedure was followed up to investigate the luminescence behavior of SCP1 to selectively detect metal ions, $\mathrm{M}=\mathrm{K}^{+}, \mathrm{Na}^{+}$, $\mathrm{Ba}^{2+}, \mathrm{Ni}^{2+}, \mathrm{Zn}^{2+}, \mathrm{Co}^{2+}, \mathrm{Mn}^{2+}, \mathrm{Cd}^{2+}, \mathrm{Fe}^{2+}, \mathrm{Pb}^{2+}$ and $\mathrm{Fe}^{3+}$ by adding $5 \mathrm{mg}$ of SCP1 in $\mathrm{CH}_{3} \mathrm{CN}$ solution which contains a constant amount of $\mathrm{M}\left(\mathrm{NO}_{3}\right)_{\mathrm{n}}\left(1 \times 10^{-3} \mathrm{~mol} . \mathrm{L}^{-1}\right)$. Then the suspension was put in ultrasonic for $10 \mathrm{~min}$ and the fluorescent intensity was measured at ambient conditions upon excitation at $360 \mathrm{~nm}[20]$.

\subsection{X -ray crystallography of single crystal of SCP1}

Crystal structure determination of SCP1 had been measured using a Kappa CCd Enraf Nonius FR 90 four circle goniometer with graphite monochromatic MoKa radiation $\{[\lambda$ MoKa $]=0.71073 \AA$ at $25 \pm 2{ }^{\circ} \mathrm{C}$. The methods used to solve the structure of $\mathbf{1}$ are performed as previously reported Rabie [4]. Structure solution, refinement by full-matrix least-squares techniques on $\mathrm{F}^{2}$ and data out-put have been completed using SHELX-97 and SIR92 programs [21]. For structure visualizations, Mercury 3.8 software was applied. Crystal data and structure refinement parameters of SCP $\mathbf{1}$ are listed in Table 1.

\section{Results and Discussion}

\subsection{X-Ray crystal structure of ${ }^{3}{ }_{\infty}[\mathrm{AgSCN}], \mathrm{SCP} 1$}


Lindqvist [22] had early studied the crystal structure of AgSCN in the space group $\mathrm{Cl} / \mathrm{c}$. The structure unit is an endless chain formed by covalent bonds from a silver atom to $\mathrm{N}$ and S-atoms of two thiocyanate groups creating a zigzag shape with bent structure. On the other hand, the crystal structure was recently refined by H.-L. Zhu indicating the formation of 3D- structure via $\mathrm{Ag}-\mathrm{S}$ bonds and weak $\mathrm{Ag}---\mathrm{Ag}$ interaction and each $\mathrm{Ag}$ atom has a $\mathrm{T}$ shape coordination environment [23]. Ag atoms, two thiocyanate ligands and four S atoms of additional SCN- groups form the fourteen-membered rings, which had been recognized as an essential unit of the $\mathrm{AgSCN}$ complex [23]. In the present discussion, we present a new overlook for the pretty crystal structure of the 3D-coordination polymer AgSCN; SCP1.

One crystal X-ray diffraction analysis of $\mathbf{1}$ confirms the monoclinic crystal system with the space group $\mathrm{C} 12 / \mathrm{c} 1$ and $\mathrm{Z}=8$, Table 1 . The asymmetric unit of SCP1 consists of the simple $\mathrm{AgSCN}$ molecule fabricated by one $\mathrm{Ag}$ atom and one thiocyanate group, as shown in Fig. 1a. The packing structure shows tetrahedral (T-4) coordination geometry around the Ag1 atom which is connected to three $\mu 3-\mathrm{S} 2$ atoms and one nitrogen end of the thiocyanate ligand which seems to be quite different than the long-recognized coordination environment of Tshape fashion [23], as can be seen in Figs. 1b,c. Thus, the SCN ligand connects four Ag atoms via $\mu 3-\mathrm{S}$ and one nitrogen atom. Three different Ag1- $\mu 3-\mathrm{S} 2$ bond lengths are observed

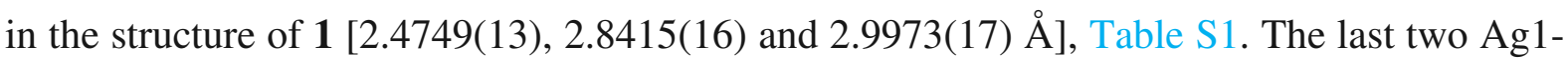
$\mu 3-\mathrm{S} 2$ bond lengths are obviously longer, while the first one is shorter than even present in molecular AgSCN (2.648 $\AA$ ) [24] and those of the prototype compounds (2.6118- 2.6543 $\mathrm{A}$ ) $[25,26]$. Also, the Ag1-N4 bond length exhibits shorter distance [2.156(4) $\AA$ ] while the S2C3 and C3-N4 bond lengths [1.660(4) and 1.166(6)], respectively, are in the range of prototype compounds.

The angles around the silver atom; 107.28(4), 97.07(3), 163.27(11) and 92.13(12) $\AA$, support the formation of distorted T-4 geometry, Table S1 and Fig. 1. Tetrahedral 
configuration can be detected by measuring the angle subtended by two planes, each encompassing the silver and two adjacent ligands. Square-planar complexes exhibits tetrahedral angle equals $0^{\circ}$. For tetrahedral complexes, the tetrahedral equals $90^{\circ}$. In SCP1 the angle of the two planes; S2Ag1S2 and N4Ag1S2 is $86.97^{\circ}$ supporting the tetrahedral topology of $\mathrm{Ag}$ atom. Two silver atoms and two sulfur atoms form rhombic mini-cycle $\left(\mathrm{Ag}_{2} \mathrm{~S}_{2}\right)$ motif via the long Ag1-S2 bond lengths while the short one connects two rings, as shown in Figs. 1b,c.

On the other hand, each rhombic mini-cycle $\left(\mathrm{Ag}_{2} \mathrm{~S}_{2}\right)$ motif connects another four $\mathrm{Ag} 2$ dimeric units (two 4-and two 8-membered rings) through Ag-S coordinate bonds to give rise to 3D-network structure, Figs 1d, 2 and 3. Interestingly, the distance between the two Ag atoms in the rhombic mini-cycle rings equals to $3.4636(17) \AA$ in addition to other two argentophilic interactions in the network structure; mainly 3.2593(17) and 3.3429(16) $\AA$. These bond lengths are obviously shorter than the van der Waals contact distance for Ag-Ag (4.26 $\AA$ ) [27] and longer than that in metal silver $(2.89 \AA)$. The mini-cycle $\left[\mathrm{Ag}_{2}\left(\mu^{3}-\mathrm{S}_{2}\right]\right.$ motifs can be considered the main building blocks of SCP1 while the network structure exhibits three different argentophilic interactions. Thus, in view of the current discussion of argentophilic interactions [28], one might suggest that the formation of a $\left[\mathrm{Ag}_{2}\left(\mu^{3}-\mathrm{S}_{2}\right]\right.$ rhomb is triggered by a weak, but non-negligible, $\mathrm{Ag}$... Ag interaction. The repeated appearance of the unusual rhombic $\left(\mathrm{Ag}_{2} \mathrm{~S}_{2}\right)$ motif causes the formation of fused polygons mainly 8 atomic $\left[\mathrm{Ag}_{2} \mathrm{~S}_{2}(\mathrm{SCN})_{2}\right], 12$ atoms $\left[\mathrm{Ag}_{4} \mathrm{~S}_{2}(\mathrm{SCN})_{2}\right], 18$ atomic $\left[\mathrm{Ag}_{5} \mathrm{~S}(\mathrm{SCN})_{4}\right]$ and 20 atomic $\left[\mathrm{Ag}_{6} \mathrm{~S}_{2}(\mathrm{SCN})_{4}\right]$ rings, as shown in Figure $\mathrm{S} 2$, creating 3D-network. The case, which raises the question of whether the mini-cycle $\left(\mathrm{Ag}_{2} \mathrm{~S}_{2}\right)$ motifs with argintophilic interactions therein are the basic cause of the existence of SCP1. The structure of SCP1 extends three-dimensionally via coordinate bonds creating stair like structure, Fig. 2. The 3-D network contains nanometer voids with average size of (34.84-58.46 nm), as can be seen in Fig 3. In addition to the strong 
coordinate bonds, the 3D-structure of SCP1 is further packed by efficient short Van der Waals contacts $(2,475-3.411 \AA)$

\subsection{Characterization and morphology of SCP1 and nanosized 2}

SCP1 and nanosized 2 phase purity are checked by measuring their powder X-ray diffractions (PXRD) which are quite similar to the simulated pattern produced by single crystal diffraction data of $\mathbf{1}$, Fig. 4. The data indicate that nanosized $\mathbf{2}$ is iso-structure to the crystal structure of SCP1. Broadening of the peaks of the PXRD pattern supports nanosized features of the particles. Moreover, the XRPD patterns of $\mathbf{2}$ were measured before and after the catalytic process displaying the same Miller indices (hkl) values which indicate high stability of $\mathbf{2}$ as a heterogeneous catalyst after photo-catalytic process, Fig. 4. Transmission Electron Microscopy (TEM) is used to measure morphology and the particle size of 2. TEM image with high magnification of $\mathbf{2}$ indicates regular spherical-like micro nano-structure exhibiting particle size in the range of $7.23-8.88 \mathrm{~nm}$, Fig. 5.

\subsection{Electronic absorption spectra, luminescence and sensing studies}

The UV spectrum of SCP1 was measured in solid state exhibiting one absorption band at $319 \mathrm{~nm}$ due to $\pi-\pi^{*}$ transition Fig. S3, with an optical band-gap of $3.88 \mathrm{eV}$ while the photo luminescent (PL) spectrum in solid state displays an intense luminescent band at 368 $\mathrm{nm}$ at $\lambda_{\mathrm{ex}}=340 \mathrm{~nm}$ corresponding to the close lying $\pi-\pi^{*}$ transition Fig. S6. The variable quenching efficiencies of different solvents and metal ions on PL spectrum of SCP1 attract considerable attention because the good ability of SCP1 to act as photo-chemical device, Figs. 6, 7. The PL band of SCP1 in MeCN shows the highest intensity meanwhile the band intensity exhibits variable quenching ability in other solvents while the most quenching efficiency was observed for NB, Fig. 6. So, the quenching character of NB can be used for determination of NB selectively where the intensity of SCP1 band decreases gradually as the NB concentration was varied from 10 to $270 \mu \mathrm{M}$. The quenching efficiency (QE) was 
calculated according to the relation; $\mathrm{QE}=\left(\mathrm{I}_{0}-\mathrm{I}\right) / \mathrm{I}_{0} \times 100 \%$. The $\mathrm{QE}$ was found to be $96.18 \%$ at NB concentration $270 \mu \mathrm{M}$ indicating the highest quenching percentage of NB against SCP1 fluorescence, Fig.S5. The Stern-Volmer constant $\left(\mathrm{K}_{\mathrm{sv}}\right)$ was calculated according to Eq (2) to be $5.55 \times 10^{3} \mathrm{M}^{-1}$ which verifies the linear behavior of the Stern-Volmer curve at low [NB], Fig. 6c. The $\mathrm{K}_{\mathrm{sv}}$ value seems similar to the reported sensors of NB [29, 30].

$$
\frac{I_{o}}{I}=1+K_{S V}[M]
$$

Io and I represent the PL intensities of SCP1 without NB and in presence of NB while quenching constant and NB concentration are represented by $\mathrm{K}_{\mathrm{sv}}$ and $[\mathrm{M}]$, respectively. The limit of detection (LOD) was calculated according to the equation [31]: $\mathrm{LOD}=3 \sigma / \mathrm{k}$ ( $\sigma$ is the standard deviation for the intensity of SCP1 in absence of NB, while k is the slope between the luminescence intensity and concentration, Fig. S6. LOD value $\left(1.723 \times 10^{-6} \mathrm{M}\right)$ is lower compared to $(5.4 \mu \mathrm{M})$ value which is accepted by the U.S. Environmental Protection Agency (EPA) $[5,32]$. The properly LOD and the effectively high QE support the reality that SCP1 is an environment friendly sensor for selective determination of NB. The mechanism of quenching of NB is mentioned utilizing the photo-induced electron switch approach [33]. Electron poor character of NB facilitates electron switch from the conduction band; $\mathrm{CB}$ of SCP1, during the excitation, to the valence band; VB of NB leading to luminescence quenching. Otherwise, an efficacious energy is conveyed via the thiocyanate $\pi$-conjugated orbitals in SCP1 to NB, inducing PL quenching as was supported by the fluorescence resonance transfer (FRET) approach [34].

Also, the PL behavior of SCP1 can be utilized for selective determination of different metal ions. The PL intensity suffers lowering according to the kind of the metal ion meanwhile $\mathrm{Fe}^{3+}$ ion exhibits the higher quenching behavior, Fig. 7. So, the selective sensing ability of SCP1 for metal ions should be used for quantitative estimation of $\mathrm{Fe}^{3+}$ ion. The PL 
intensity of SCP1 suffers gradual decrease upon increasing $\mathrm{Fe}^{3+}$ ion concentration in the range of $10-330 \mu \mathrm{M}$, Fig.7b. The plot of Stern-Volmer is linear up to $45 \mu \mathrm{M}$ suffering deviation from being linear at higher concentrations, Fig. 7c. QE, LOD and $\mathrm{K}_{\mathrm{sv}}$ for $\mathrm{Fe}^{3+}$ ion are estimated giving the values of $97.57 \%, 4.53 \mu \mathrm{M}$ and $4.08 \times 10^{-3} \mathrm{M}^{-1}$, respectively, which are close to the reported values of the prototype compounds, Figs S7, S8 [5, 35]. SCP1 can be considered as an excellent PL sensor for quantitative determination of $\mathrm{Fe}^{3+}$ ion because low LOD and high QE. The mechanism of quenching phenomenon of $\mathrm{Fe}^{3+}$ ion is discussed according to the FRET approach where PL quenching resulted via the interfere of the electronic transition band of $\mathrm{Fe}^{3+}$ ion with the PL band of SCP1, as well as the effect of Lewis acidic $\mathrm{Fe}^{3+}$ cation on SCP1 resulting PL quenching [36].

\subsection{Catalytic and photocatalytic degradation studies of nanosized 2}

The photocatalytic activity of nanosized 2 was investigated in the presence of $\mathrm{H}_{2} \mathrm{O}_{2}$ as eco-friendly nature using Acid Blue 92 (AB-92) and Eosin-Y (EO-Y) dyes as target pollutants. (AB-92) and (EO-Y) dyes exhibit high stability leading to hard decomposition in waste streams. The mineralization processes were carried out by measuring the absorption spectra of (AB-92) and (EO-Y) at 560 and $514 \mathrm{~nm}$, respectively. The visible spectra of control reactions in presence of $A B-92$ and EO-Y dyes and $\mathrm{H}_{2} \mathrm{O}_{2}$ at ambient conditions exhibit D\% $=10.89 \%$ and $10.02 \%$ within 160 min, respectively, Figs. S9, S10. In this case, there is no worth mineralization of the tested dyes without using a catalyst. Interestingly, the spectra of AB-92 and EO-Y dyes suffer obvious gradual decrease of the peaks intensity in the presence of the nanosized 2 and $\mathrm{H}_{2} \mathrm{O}_{2}$ while on subjecting UV-light or ultrasonic irradiation to the reactors, the peak intensities decrease dramatically during short times, Table 2 . The rate of mineralization as well as the removal efficiency of the tested dyes exhibit notable increase according to the order ultrasonic irradiation $>$ UV-light $>$ room temperature [in case of AB-92 dye, $\mathrm{D} \%=97.89$ (within $35 \mathrm{~min}$ ), 89.47 (within $105 \mathrm{~min}$ ) and 89.47 (within 160 
min),respectively, Fig. 8 and for EO-Y dye, D\%= 99.36 (within 48 min), 89.24 (within 105 min) and 81.04 (within $145 \mathrm{~min}$ ), Fig. 9, respectively], Table 2. The mineralization efficiency of nanosized 2 for AB-92 and EO-Y dyes reaches a highest value on using $\mathrm{H}_{2} \mathrm{O}_{2}$ and ultrasonic irradiation within very short time. The clear enhancements of photocatalytic degradation rates of the dyes support the energetic character of $\mathbf{2}$ for complete degradation of the tested dyes.

First order kinetic equations are applied to study the degradation reaction with respect to $[\text { dye }]_{\mathrm{o}}$ concentration where the $\left[\mathrm{H}_{2} \mathrm{O}_{2}\right]_{\mathrm{o}}$ initial concentration was excess 10 -fold more than the $[\text { dye }]_{\mathrm{o}}$. kobs (observed rate constant) was calculated according to Eq. (3).

$$
\ln \frac{A_{o}}{A_{t}}=K t
$$

$\mathrm{A}_{\mathrm{o}}$ and $\mathrm{A}_{\mathrm{t}}$ are the absorbance of dyes at time $=0$ and $\mathrm{t}$, respectively. The linear relationships support that the degradation reaction obeys pseudo first order rate relative to [dye $]_{\mathrm{o}}$, Table 2 and Figs. S11, 12. The specific rate constant $(\mathrm{k})$ was calculated using equation $\mathrm{k}=$ $\mathrm{k}_{\mathrm{obs}} /\left[\mathrm{H}_{2} \mathrm{O}_{2}\right]_{\mathrm{o}}$, Table 2 .

To address reusing of the catalyst, it was cleaned by deionized $\mathrm{H}_{2} \mathrm{O}$ and dried at the end of the first use and then after a new experiment was carried out. The catalyst $\mathbf{2}$ exhibits the same catalytic activity for two cycles of the dye oxidation under ultrasonic irradiation, Fig. 10. After the second recycle, the time and efficiency of degradation remain steady as those at typical conditions while the degradation efficiency decreases slightly till the fourth cycle. The experimental PXRD patterns of nanosized 2 before and after mineralization reactions were recorded showing very good similarity compared to the simulated XRD pattern of SCP1 indicating that the sample of nanosized $\mathbf{2}$ has the same structure of the single crystals preserving its phase purity and stability as heterogeneous catalyst, Fig. 4. These results are further confirmed by elemental analysis. 


\subsection{Active species and mechanism of the catalytic and photo- catalytic processes}

Identification of the type of the active species created in the EO-Y/ nanosized $2 / \mathrm{H}_{2} \mathrm{O}_{2}$ system, mainly $\bullet \mathrm{OH}, \mathrm{h}+$, and $\mathrm{O}_{2}{ }^{-}$, requires some investigations using isopropanol (IPA), ammonium oxalate (AO), or benzoquinone (BQ) as scavengers, respectively. IPA, AO or BQ added to EO-Y / nanosized $2 / \mathrm{H}_{2} \mathrm{O}_{2}$ system decreased the degradation efficiency of EO to $76.20 \%$ in $140 \mathrm{~min}, 66.20 \%$ within $140 \mathrm{~min}$ and $32.50 \%$ within $140 \mathrm{~min}$, respectively, Table 2, Figs. S13-16. In this case, the $\bullet \mathrm{OH}$ active species have been quenched significantly by IPA while $\mathrm{h}+$, and $\mathrm{O}_{2}{ }^{-}$active species exhibit, relatively lower quenching effect by $\mathrm{AO}$ and $\mathrm{BQ}$, respectively. The case which indicates that the catalytic process is highly affected by the formation rate of the $\bullet \mathrm{OH}$ radicals than the $\mathrm{h}+$, and $\mathrm{O}_{2}{ }^{\cdot-}$ active species.

For further support of the activity of $\bullet \mathrm{OH}$ radicals terephthalic acid disodium salt (TANa) PL probing technology was applied which linked selectively with hydroxyl radicals to produce the fluorescent 2-hydroxy terephthalic acid (HTA) [37], The fluorescence intensity of catalyst $2 / \mathrm{H}_{2} \mathrm{O}_{2} /$ TANa component elevated rapidly to 950 within 56 min supporting that $\bullet \mathrm{OH}$ species are really produced in solution, Fig. 12a. Consequently, when the tested dye and TANa are both put to the solution, the intensity of fluorescence spectra decreased apparently reaching only to 230 within $64 \mathrm{~min}$, Fig. $12 \mathrm{~b}$. It is obvious that some of the $\cdot \mathrm{OH}$ radicals are subjected to fast oxidation to the dye, as rapid fading of the dye color was also noticed during recording the spectra. For that, consulting the data, a mechanism can be postulated which involves an interaction between $\mathrm{H}_{2} \mathrm{O}_{2}$ and the tested catalyst with generating highly active oxidizing species, mainly $\bullet \mathrm{OH}$ radicals.

$$
\begin{aligned}
& \mathrm{H}_{2} \mathrm{O}_{2}+\left[\text { nanosized 2] } \rightarrow \bullet \mathrm{OH}+\mathrm{OH}^{-}+[\text {nanosized 2 }]^{+}\right. \\
& \mathrm{OH}^{-}+[\text {nanosized 2 }]^{+} \rightarrow[\text { nanosized } 2(\mathrm{OH})] \leftrightarrow[\text { nanosized } 2]+\bullet \mathrm{OH}
\end{aligned}
$$

The hydroxyl radicals react with the tested dyes to propagate the reaction to intermediates leading to degradation products. 
- $\mathrm{OH}+$ dye $\rightarrow$ Intermediates

Intermediates $\rightarrow$ oxidation products $+\mathrm{CO}_{2}+\mathrm{H}_{2} \mathrm{O}$

Other reactions are also possible as indicated by Eqs. ( 8 and 9).

$\bullet \mathrm{OH}+\bullet \mathrm{OH} \rightarrow \mathrm{H}_{2} \mathrm{O}_{2}$

- $\mathrm{OH}+\mathrm{H}_{2} \mathrm{O}_{2} \rightarrow \bullet \mathrm{OOH}^{-}+\mathrm{H}_{2} \mathrm{O}$

The peroxide radicals $\left(\mathrm{HO}_{2}{ }^{-}\right)$can further oxidize other species present in the solution [38].

- $\mathrm{OOH}^{-}+\left[\right.$nanosized 2] $\rightarrow \mathrm{O}_{2}+\left[\right.$ nanosized 2] $++\mathrm{H}^{+}$

In addition to that the degradation efficiency of the dye is enhanced at normal conditions upon utilizing UV- light or ultrasonic radiation where irradiation improved producing of $\bullet \mathrm{OH}$ radicals. $\mathrm{H}_{2} \mathrm{O}_{2}$ as a green oxidant in addition to the use of light irradiation can create $\cdot \mathrm{OH}$ radicals as indicated in $\mathrm{Eq}(11)$ :

$h v+\mathrm{H}_{2} \mathrm{O}_{2} \rightarrow 2 \cdot \mathrm{OH}$

The high occupied molecular orbital containing electrons of the nanosized $\mathbf{2}$ defined as the valence band (VB) can be excited under the UV-radiation to the lowest unoccupied orbital the conduction band (CB). The electron transfer causes production of a similar quantity of positive holes $\left(\mathrm{h}^{+}\right)$which leave the VB, Eq. (12). The $\bullet \mathrm{OH}$ radicals are formed via the addition of $\mathrm{H}^{+}$to $\mathrm{O}_{2}{ }^{--}$produced by the reduction of $\mathrm{O}_{2}$ by $\mathrm{e}^{-}$, Eqs (13-16) and the oxidation of $\mathrm{H}_{2} \mathrm{O}$ by $\mathrm{h}^{+}$creating $\bullet \mathrm{OH}$, Eq (17). The $\bullet \mathrm{OH}$ radicals oxidize the IC dye converting them to degradation products, $\mathrm{CO}_{2}$ and $\mathrm{H}_{2} \mathrm{O}$, Eqs. $(6,7)$.

$$
\begin{aligned}
& \text { catalyst2 }+h v \rightarrow h^{+}{ }_{V B}+e^{-}{ }_{C B} \\
& e^{-}{ }_{C B}+\mathrm{O}_{2} \rightarrow \bullet \mathrm{O}_{2}^{-} \\
& \bullet \mathrm{O}_{2}^{-}+\mathrm{H}^{+} \rightarrow \bullet \mathrm{OOH} \\
& 2 \bullet \mathrm{OOH} \rightarrow \mathrm{O}_{2}+\mathrm{H}_{2} \mathrm{O}_{2} \\
& \mathrm{H}_{2} \mathrm{O}_{2}+h v \rightarrow 2 \cdot \mathrm{OH}
\end{aligned}
$$




$$
h^{+}{ }_{V B}+H_{2} \mathrm{O} \rightarrow \bullet O H+H^{+}
$$

On the other hand, $\mathrm{H}_{2} \mathrm{O}$ splits by ultrasonic irradiation [ )))] as shown in Eqs (18-20).

$$
\mathrm{H}_{2} \mathrm{O}_{2} \stackrel{)))}{\rightarrow} \cdot \mathrm{H}+\bullet \mathrm{OH}
$$

$\bullet \mathrm{OH}+\bullet \mathrm{OH} \rightarrow \mathrm{H}_{2}+\mathrm{O}_{2}$

- $\mathrm{H}+\mathrm{O}_{2} \rightarrow \bullet \mathrm{OOH}$

The ultrasonic radiation assisted decomposition of $\mathrm{H}_{2} \mathrm{O}_{2}$ in the reaction medium according to Eqs (21-23).

$$
\begin{aligned}
& \mathrm{H}_{2} \mathrm{O}_{2} \stackrel{)}{\rightarrow} 2 \cdot \mathrm{OH} \\
& e_{\mathrm{CB}}{ }^{-}+\mathrm{H}_{2} \mathrm{O}_{2} \rightarrow \bullet \mathrm{OH}+\mathrm{OH}^{-} \\
& \bullet \mathrm{O}_{2}^{-}+\mathrm{H}_{2} \mathrm{O}_{2} \rightarrow \bullet \mathrm{OH}+\mathrm{OH}^{-}+\mathrm{O}_{2}
\end{aligned}
$$

At the end, the $\cdot \mathrm{OH}$ radicals oxidize the tested dyes producing final oxidation products according to Eqs. (6 and 7).

\section{Conclusion}

The structures of SCP1 and nanosized 2 represent single crystals and micro nano-particles prepared by self-assembly and green method of ultrasonic radiation, respectively. 3Dframework is constructed via coordinate bonds and the unique mini-cycle $\left(\mathrm{Ag}_{2} \mathrm{~S}_{2}\right)$ motif. The fluorescence behavior and high quenching efficiency of SCP1 for $\mathrm{Fe}^{3+}$ ions and NB lead to be considered as a good and efficient candidate for selective detection of $\mathrm{Fe}^{3+}$ ion in presence of other metal ions and explosives. The UV-and ultrasonic radiation-catalytic processes presented in this study proved to be propitious devices for the elimination of nonbiodegradable dyes like AB and EO dyes. The tested catalyst $\mathbf{2}$ acts as heterogeneous micro nano-catalyst for the efficient elimination of dyes in wastewater within relatively short times 
mainly via $\bullet \mathrm{OH}$ radicals according to (TANa) PL probing technology. The tested dyes follow the Fenton process which can be considered a powerful AOP, due to the fact that AOP does not need special equipment or energy input and capable to oxidize various organic pollutants utilizing the high oxidation potential of the $\bullet \mathrm{OH}$ radical. 


\section{References}

[1] J.-Q. Liu, Z.-D. Luo, Y. Pan, A.K. Singh, M. Trivedi, A. Kumar, Recent developments in luminescent coordination polymers: Designing strategies, sensing application and theoretical evidences, Coordination chemistry reviews, 406 (2020) 213145.

[2] Y. Yuan, Y.-W. Xiao, X.-S. Yan, S.-X. Wu, H. Luo, J.-B. Lin, Z. Li, Y.-B. Jiang, Supramolecular chirality of coordination polymers of $\mathrm{Ag}+$ with a chiral thiol ligand that bears a $\beta$-turn structure, Chemical communications, 55 (2019) 12849-12852.

[3] C. Zhang, C. Zhang, Y. Xie, J.W. Su, X. He, J.D. Demaree, M.H. Griep, J.L. Atwood, J. Lin, A Supramolecular Coordination-Polymer-Derived Electrocatalyst for the Oxygen Evolution Reaction, Chemistry-A European Journal, 25 (2019) 4036-4039.

[4] S.E.d.H. Etaiw, H. Marie, E.M. Shalaby, R.S. Farag, F.A. Elsharqawy, Sensing and photocatalytic properties of nanosized $\mathrm{Cu}$ (I) $\mathrm{CN}$ organotin supramolecular coordination polymer based on pyrazine, Applied Organometallic Chemistry, 33 (2019) e5114.

[5] S.E.-d.H. Etaiw, H. Marie, Sonochemical nanostructure of Mn (II) supramolecular complex: X-ray structure, sensing and photocatalytic properties, Sensors and Actuators B: Chemical, 290 (2019) 631-639.

[6] S.E.H. Etaiw, R.S. Farag, H. Marie, F.A. Elsharqawy, Crystal structure and sonochemical nanosized synthesizing of diaquo-bis-(pyrazine-2-carboxylato) copper (II) complex: Sensing and photocatalytic activity, Solid State Sciences, (2020) 106160.

[7] M. Elkelawy, S.E.-d.H. Etaiw, H.A.-E. Bastawissi, H. Marie, A. Elbanna, H. Panchal, K. Sadasivuni, H. Bhargav, Study of diesel-biodiesel blends combustion and emission characteristics in a CI engine by adding nanoparticles of Mn (II) supramolecular complex, Atmospheric Pollution Research, 11 (2020) 117-128.

[8] S. Bera, A. Chowdhury, K. Sarkar, P. Dastidar, Design and Synthesis of ZnIICoordination Polymers Anchored with NSAIDs: Metallovesicle Formation and Multi-drug Delivery, Chemistry-An Asian Journal, 15 (2020) 503-510.

[9] Y.-S. Shi, Q.-Q. Xiao, L. Fu, G.H. Cui, Three water-stable luminescent two-dimensional CdII-based coordination polymers as sensors for highly sensitive and selective detection of Cr2O72-and CrO42-anions, CrystEngComm, (2020).

[10] V. Remya, M. Kurian, Synthesis and catalytic applications of metal-organic frameworks: a review on recent literature, International Nano Letters, 9 (2019) 17-29. 
[11] Y.-S. Shi, Z.-C. Hao, C. Han, G.-H. Cui, Synthesis, crystal structures, and luminescence sensing properties of two metal complexes containing bis (thiabendazole) moieties, Journal of Molecular Structure, 1201 (2020) 127218.

[12] Q.-Q. Tu, L.-L. Ren, Y.-Y. Cui, A.-L. Cheng, E.-Q. Gao, Assembly of four new cobalt coordination polymers modulated by N-coligands: sensitive and selective sensing of nitroaromatics, Fe 3+ and Cr 2 O 7 2- in water, CrystEngComm, 22 (2020) 1789-1801.

[13] C.-X. Wang, Y.-P. Xia, Z.-Q. Yao, J. Xu, Z. Chang, X.-H. Bu, Two luminescent coordination polymers as highly selective and sensitive chemosensors for Cr VI-anions in aqueous medium, Dalton Transactions, 48 (2019) 387-394.

[14] H. Zhu, Y.-H. Li, Q.-Q. Xiao, G.-H. Cui, Three luminescent Cd (II) coordination polymers containing aromatic dicarboxylate and flexible bis (benzimidazole) ligands as highly sensitive and selective sensors for detection of $\mathrm{Cr} 2 \mathrm{O} 72-$ oxoanions in water, Polyhedron, (2020) 114648.

[15] H. Zhu, L. Fu, D. Liu, Y.-H. Li, G.-Y. Dong, Three water-stable luminescent Zn (II) coordination polymers for highly sensitive and selective sensing of acetylacetone and $\mathrm{Fe} 3+$ ions, Journal of Solid State Chemistry, 286 (2020) 121265.

[16] S.A. Baudron, Ag (I)- $\pi$ interactions with pyrrolic derivatives, Coordination chemistry reviews, 380 (2019) 318-329.

[17] T.A. Hegde, A. Dutta, V. Gandhiraj, Review on growth and characterization of nonlinear optical organometallic thiocyanate crystals, International Journal of Engineering and Technology Innovation, 9 (2019) 257.

[18] S.E.-d.H. Etaiw, H. Marie, Ultrasonic synthesis of $1 \mathrm{D}-\mathrm{Zn}$ (II) and La (III) supramolecular coordination polymers nanoparticles, fluorescence, sensing and photocatalytic property, Journal of Photochemistry and Photobiology A: Chemistry, 364 (2018) 478-491.

[19] S.E.-d.H. Etaiw, H. Marie, 3D-Supramolecular Coordination Polymer Nanoparticles Based on Cd (II) and Mixed Ligands: Single Crystal X-Ray Structure, Luminescence and Photocatalytic Properties, Journal of Inorganic and Organometallic Polymers and Materials, 28 (2018) 508-518.

[20] D. Das, K. Biradha, Luminescent coordination polymers of naphthalene based diamide with rigid and flexible dicarboxylates: sensing of nitro explosives, Fe (III) ion, and dyes, Crystal Growth \& Design, 18 (2018) 3683-3692.

[21] G.M. Sheldrick, Acta Crystallogr., Sect. A, A: Found. Crystallogr, 64 (2008) 112. 
[22] I. Lindqvist, On the crystal structure of silver thiocyanate, Acta Crystallographica, 10 (1957) 29-32.

[23] H.-L. Zhu, X.-M. Zhang, Q. Yu, D.-Q. Wang, Crystal structure of aqua-bis (4nitrobenzoato) disilver (I), Ag2 (C7H4NO4) 2 (H2O), Zeitschrift für Kristallographie-New Crystal Structures, 218 (2003) 263-264.

[24] F.A. Cotton, G. Wilkinson, C.A. Murillo, M. Bochmann, R. Grimes, Advanced inorganic chemistry, Wiley New York, 1988.

[25] X. Yin, S. Meng, J. Xie, Heterobimetallic $\mathrm{Mg}-\mathrm{Ag}$ coordination polymer with luminescence and 2, 4, 6-Trinitrophenol sensing properties, Polyhedron, 139 (2018) 262-266. [26] S.E.-d.H. Etaiw, D.M.A. El-Aziz, M.S. Ibrahim, A.S.B. El-din, Synthesis and crystal structures of three novel coordination polymers constructed from Ag (I) thiocyanate and nitrogen donor ligands, Polyhedron, 28 (2009) 1001-1009.

[27] S.S. Batsanov, Van der Waals radii of elements, Inorganic materials, 37 (2001) 871-885. [28] S.E.-d.H. Etaiw, D.M.A. El-Aziz, A.S.B. El-din, Self-assembly of supramolecular coordination polymers constructed from $\mathrm{AgCN}$ and bipodal spacers, Polyhedron, 28 (2009) 873-882.

[29] J.J. Przybyla, R.L. LaDuca, Nitrobenzene-detection cadmium thiophenedicarboxylate coordination polymers with flexible dipyridylamide ligands and diverse topologies, Inorganica Chimica Acta, 486 (2019) 314-323.

[30] Y.-Q. Zhang, V.A. Blatov, T.-R. Zheng, C.-H. Yang, L.-L. Qian, K. Li, B.-L. Li, B. Wu, A luminescent zinc (II) coordination polymer with unusual $(3,4,4)$-coordinated selfcatenated 3D network for selective detection of nitroaromatics and ferric and chromate ions: a versatile luminescent sensor, Dalton Transactions, 47 (2018) 6189-6198.

[31] A. Buragohain, M. Yousufuddin, M. Sarma, S. Biswas, 3D luminescent amidefunctionalized cadmium tetrazolate framework for selective detection of 2, 4, 6trinitrophenol, Crystal Growth \& Design, 16 (2016) 842-851.

[32] U.D.o. Health, H. Services, Public Health Service, Agency for Toxic Substances and Disease Registry, Toxicological profile for copper, (2004).

[33] W.-X. Li, H.-X. Li, H.-Y. Li, M.-M. Chen, Y.-X. Shi, J.-P. Lang, 1, 4-Bis (2-(pyridin-4yl) vinyl) naphthalene and its zinc (II) coordination polymers: synthesis, structural characterization, and selective luminescent sensing of mercury (II) ion, Crystal Growth \& Design, 17 (2017) 3948-3959.

[34] Y. Rachuri, B. Parmar, K.K. Bisht, E. Suresh, Mixed ligand two dimensional Cd (II)/Ni (II) metal organic frameworks containing dicarboxylate and tripodal $\mathrm{N}$-donor ligands: $\mathrm{Cd}$ (II) 
MOF is an efficient luminescent sensor for detection of picric acid in aqueous media, Dalton Transactions, 45 (2016) 7881-7892.

[35] X. Han, J. Yang, Y.-Y. Liu, J.-F. Ma, Nine coordination polymers assembled with a novel resorcin [4] arene tetracarboxylic acid: Selective luminescent sensing of acetone and Fe3+ ion, Dyes and Pigments, 160 (2019) 492-500.

[36] X.R. Wang, X.Z. Wang, Y. Li, K. Liu, S.X. Liu, J. Du, Z. Huang, Y. Luo, J.Z. Huo, X.X. Wu, Sonochemical synthesis of a multi-responsive regenerable water-stable zinc (II) fluorescent probe for highly selective, sensitive and real-time sensing of benzaldehyde, ferric ion and PH, Ultrasonics sonochemistry, 44 (2018) 340-349.

[37] J. Yu, Q. Xiang, M. Zhou, Preparation, characterization and visible-lightdriven photocatalytic activity of Fe-doped titania nanorods and first-principles study for electronic structures, Applied Catalysis B: Environmental, 90 (2009) 595-602.

[38] S.E.-d.H. Etaiw, H. Marie, Two new Ni (II) supramolecular complexes based on ethyl isonicotinate and ethyl nicotinate for removal of acid blue 92 dye, Solid State Sciences, 77 (2018) 5-13 
Table 1 Crystal data and structures refinement parameters of the SCP1.

\begin{tabular}{|c|c|}
\hline Chemical formula & $\mathrm{C}_{1} \mathrm{Ag}_{1} \mathrm{~N}_{1} \mathrm{~S}_{1}$ \\
\hline Formula weight & 165.95 \\
\hline Crystal system & Monoclinic \\
\hline Space group & $\mathrm{C} 12 / \mathrm{c} 1$ \\
\hline$a(\AA)$ & $8.815(5)$ \\
\hline$b(\AA)$ & $7.988(5)$ \\
\hline$c(\AA)$ & $8.207(4)$ \\
\hline$\alpha\left(^{\circ}\right)$ & 90 \\
\hline$\beta\left(^{\circ}\right)$ & $93.766(16)$ \\
\hline$\gamma\left(\left(^{\circ}\right)\right.$ & 90 \\
\hline$V\left(\AA^{3}\right)$ & $576.7(5)$ \\
\hline$Z$ & 8 \\
\hline$D_{\text {calcd }}\left(\mathrm{g} / \mathrm{cm}^{3}\right)$ & 3.82 \\
\hline Absorption coefficient, $\mathrm{mm}^{-1}$ & 7.385 \\
\hline$F(000)$ & 608 \\
\hline Crystal size, $\mathrm{mm}$ & $0.040 \times 0.070 \times 0.090$ \\
\hline$\theta$ range, deg & $1.213-34.630$ \\
\hline Index range $h, k, l$ & $-23 / 23,0 / 17,0 / 46$ \\
\hline $\mathrm{R}_{\text {int }}$ & 0.037 \\
\hline Data/restraint/parameters & $3980 / 0 / 38$ \\
\hline Goodness of fit on $F^{2}$ & 1.0882 \\
\hline Final $\mathrm{R}_{1}, w \mathrm{R}_{2}(I>2 \sigma(I))$ & $0.0432,0.1400$ \\
\hline$\Delta \rho_{\max }, \mathrm{e} \AA^{-3}, \Delta \rho_{\min }, \mathrm{e} \AA^{-3}$ & $1.21,-1.75$ \\
\hline CCDC NO & 2007622 \\
\hline
\end{tabular}


Table 2 The kinetic parameters for the photodegradation of AB-92 and EO-Y dyes using nanosized 2

\begin{tabular}{|c|c|c|c|c|c|}
\hline Catalyst & Condition & $\begin{array}{l}\text { Irradiation } \\
\text { Time (min) }\end{array}$ & Removal \% & $\mathrm{K}\left(\mathbf{m i n}^{-1}\right)$ & $\overline{\mathbf{R}^{2}}$ \\
\hline Non & $\mathrm{AB}-92+\mathrm{H}_{2} \mathrm{O}_{2}$ & 160 & 11.89 & $6.44 \times 10^{-4}$ & 0.984 \\
\hline Nanosized 2 & $\mathrm{AB}-92 /$ Room $+\mathrm{H}_{2} \mathrm{O}_{2}$ & 160 & 83.33 & 0.0097 & 0.994 \\
\hline Nanosized 2 & $\mathrm{AB}-92 / \mathrm{UV}+\mathrm{H}_{2} \mathrm{O}_{2}$ & 105 & 83.43 & 0.0176 & 0.997 \\
\hline Nanosized 2 & AB-92 /Sonication $+\mathrm{H}_{2} \mathrm{O}_{2}$ & 35 & 92.71 & 0.0595 & 0.968 \\
\hline Non & $\mathrm{EO}-\mathrm{Y}+\mathrm{H}_{2} \mathrm{O}_{2}$ & 160 & 10.02 & $4.74 \times 10^{-4}$ & 0.981 \\
\hline Nanosized 2 & EO-Y / Room $+\mathrm{H}_{2} \mathrm{O}_{2}$ & 145 & 73.25 & 0.00932 & 0.998 \\
\hline Nanosized 2 & $\mathrm{EO}-\mathrm{Y} / \mathrm{UV}+\mathrm{H}_{2} \mathrm{O}_{2}$ & 105 & 84.2 & 0.0171 & 0.996 \\
\hline Nanosized 2 & EO-Y / Sonication $+\mathrm{H}_{2} \mathrm{O}_{2}$ & 38 & 93.76 & 0.0573 & 0.954 \\
\hline Nanosized 1 & $\mathrm{EO}-\mathrm{Y} / \mathrm{UV}+\mathrm{H}_{2} \mathrm{O}_{2}+\mathrm{AO}$ & 140 & 68.46 & 0.0095 & 0.971 \\
\hline Nanosized 1 & $\mathrm{EO}-\mathrm{Y} / \mathrm{UV}+\mathrm{H}_{2} \mathrm{O}_{2}+\mathrm{BQ}$ & 140 & 56.96 & 0.0063 & 0.992 \\
\hline Nanosized 1 & EO-Y / UV $+\mathrm{H}_{2} \mathrm{O}_{2}+\mathrm{IPA}$ & 140 & 29.33 & 0.0027 & 0.988 \\
\hline
\end{tabular}




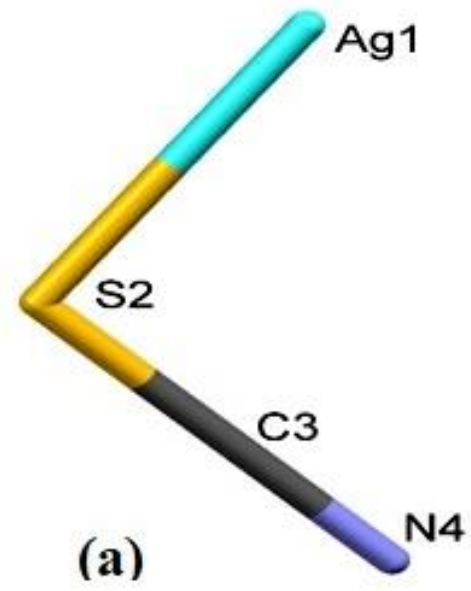

(a)

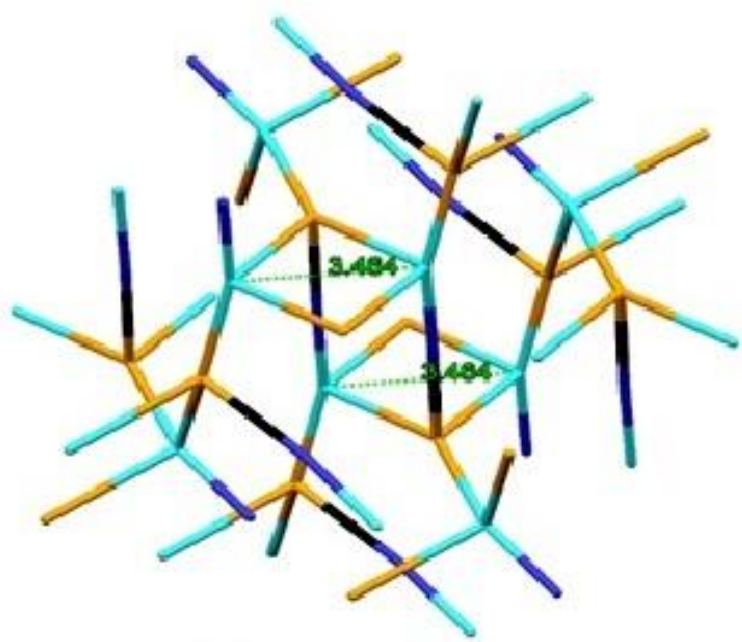

(c)

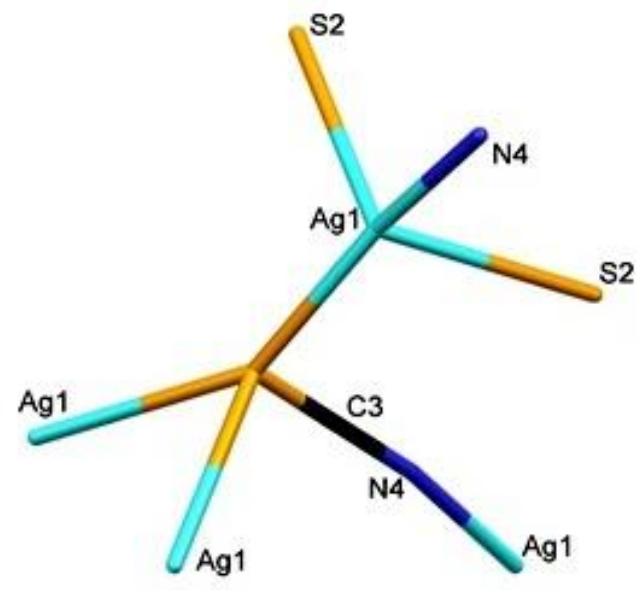

(b)

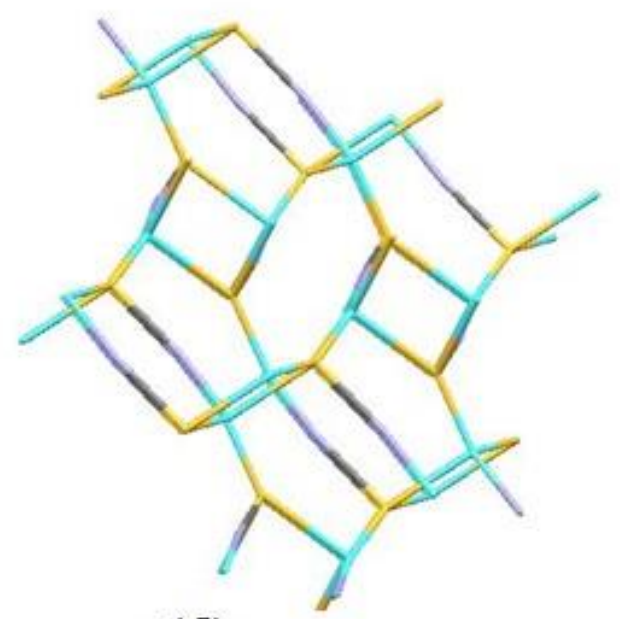

(d)

Figure 1 (a)Asymmetric unit of $\mathrm{AgSCN}$ showing atom labeling scheme, (b) view of tetrahedral geometry of $\mathrm{AgSCN}$ along a-axis, (c) packing structure showing the complex connections via quadro minicycle $\left(\mathrm{Ag}_{2} \mathrm{~S}_{2}\right)$ motifs extending the structure three dimensionally, (d) view of the distorted polygonal rings 


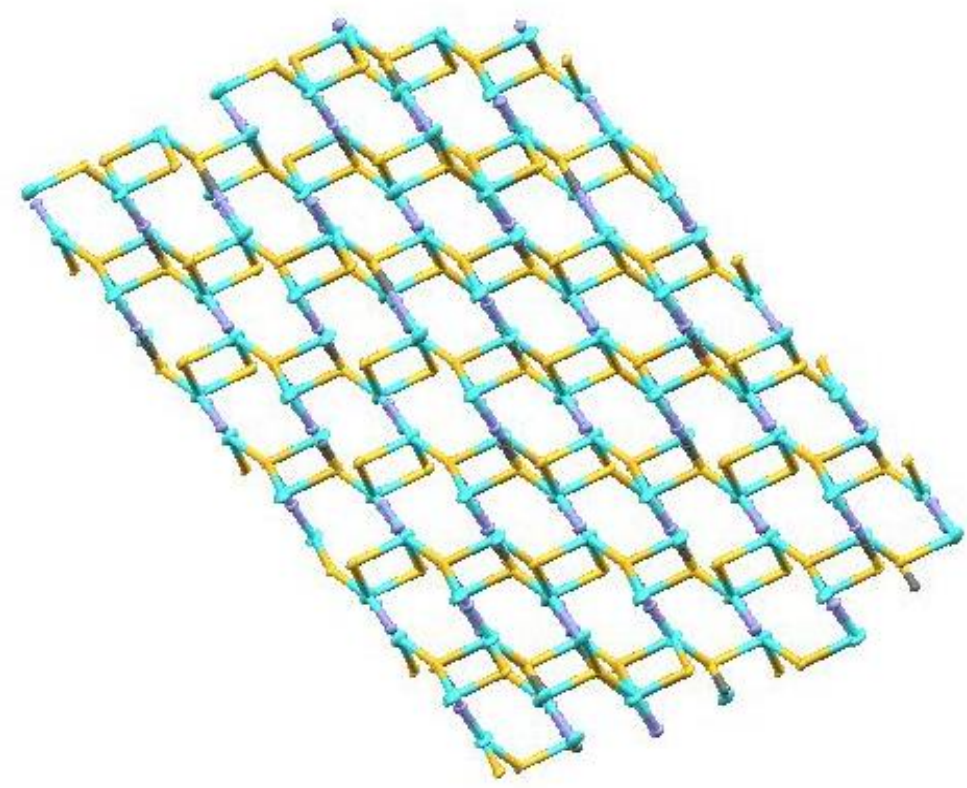

Figure 2 View of the 3D-network structure of SCP1 showing the stair construction along the b-axis

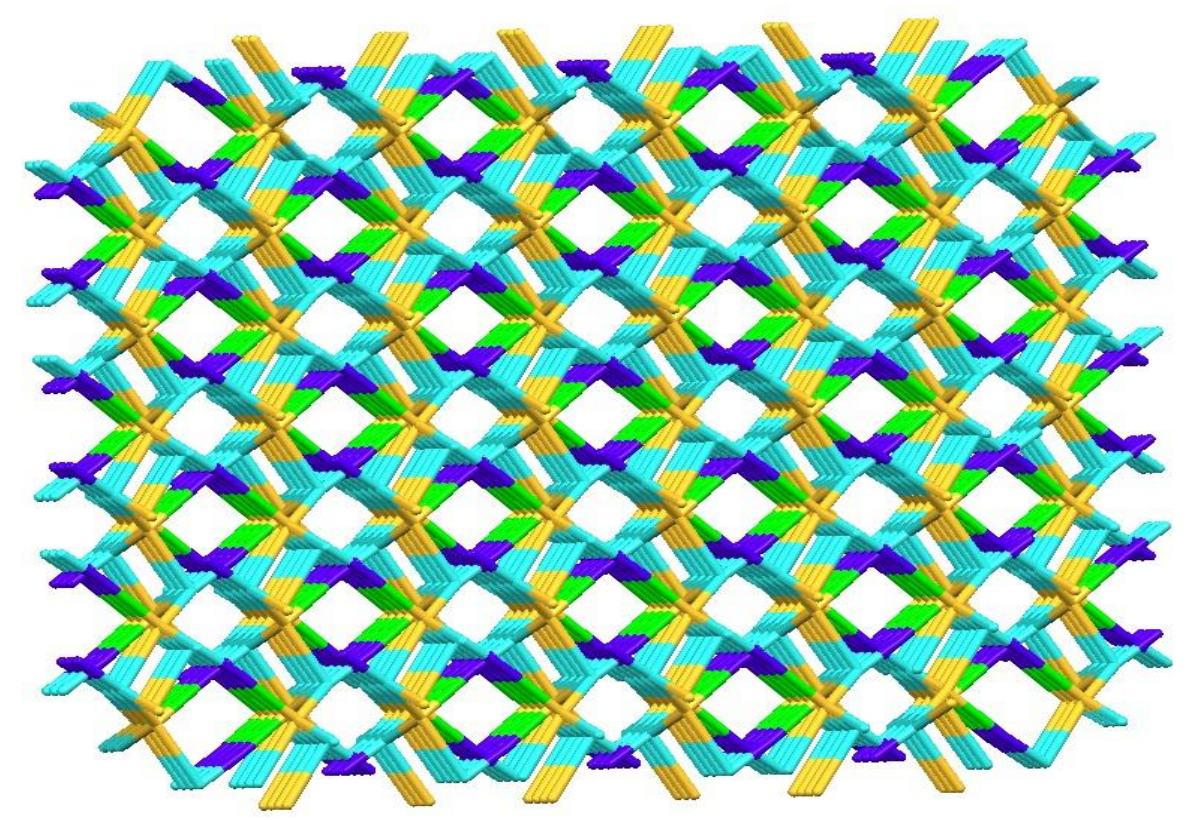

Figure 3 Visualization of the 3D-network structure of SCP1 down the projection of the a axis showing the pore structure 


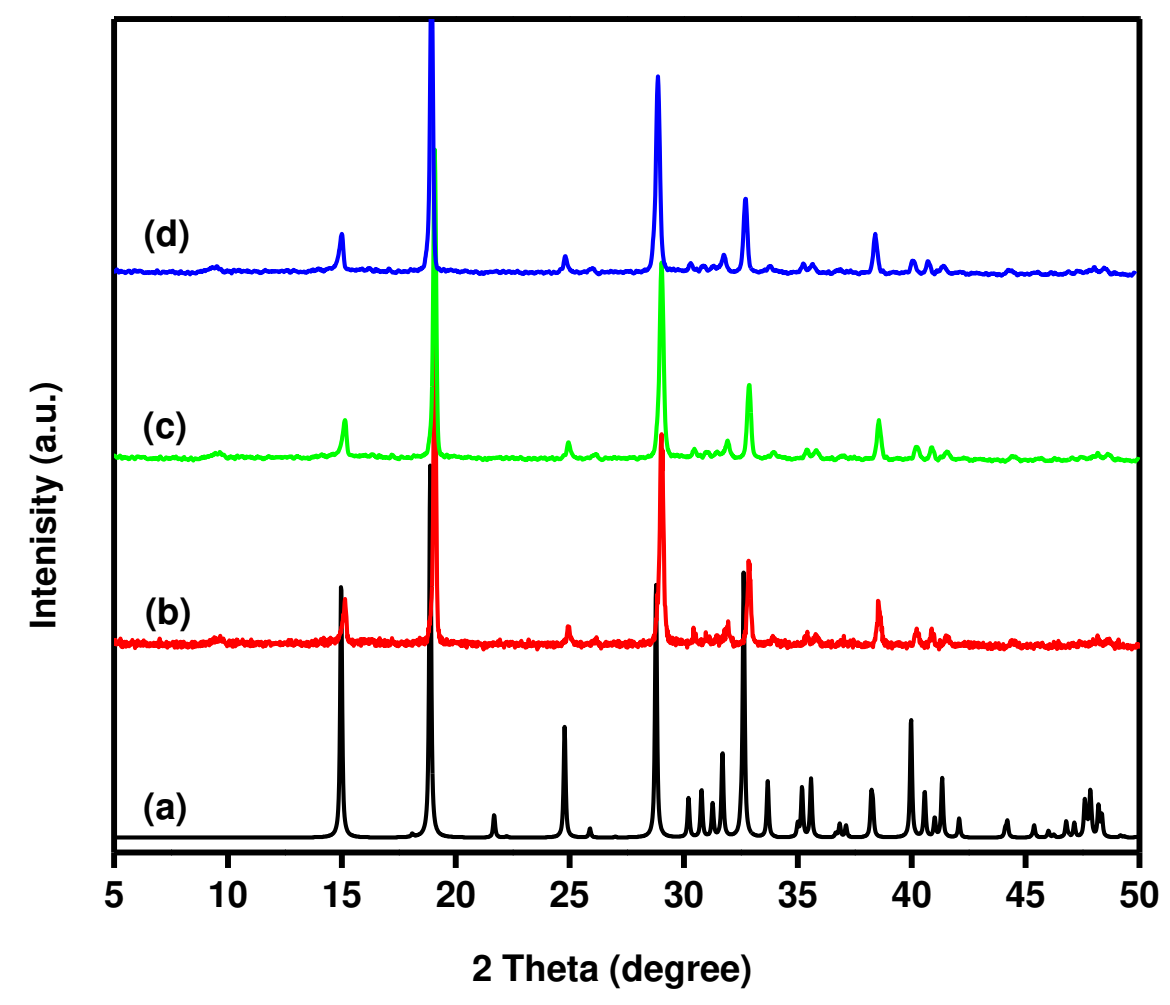

Figure 4 PXRD patterns: (a) simulated pattern based on single crystal data of SCP1; (b) SCP1 as synthesized by self-assembly method; (c) nanosized $\mathbf{2}$ before degradation process;(d) nanosized $\mathbf{2}$ after degradation process 


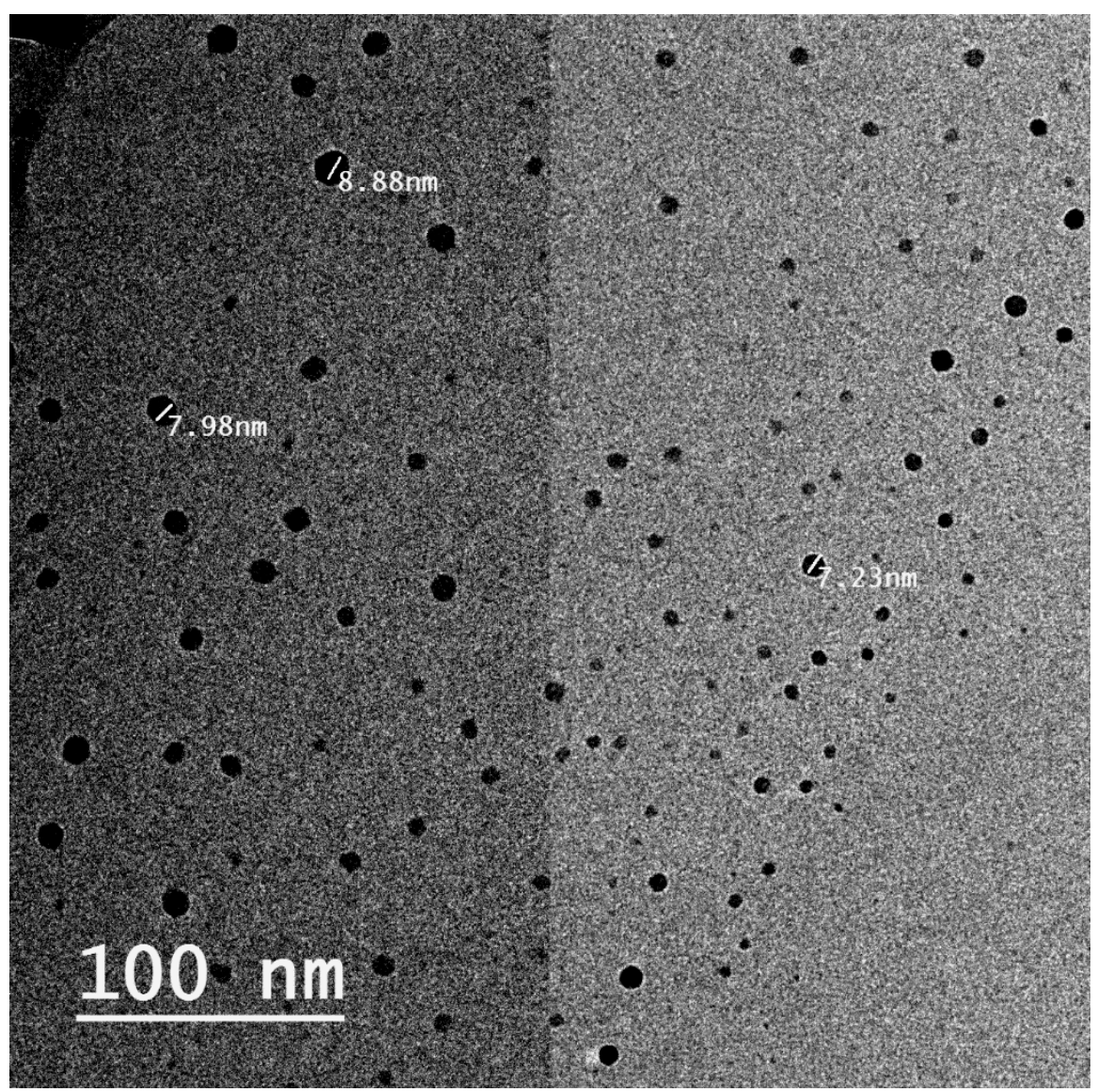

Figure 5 The TEM image of 2 showing the nanosized particles 

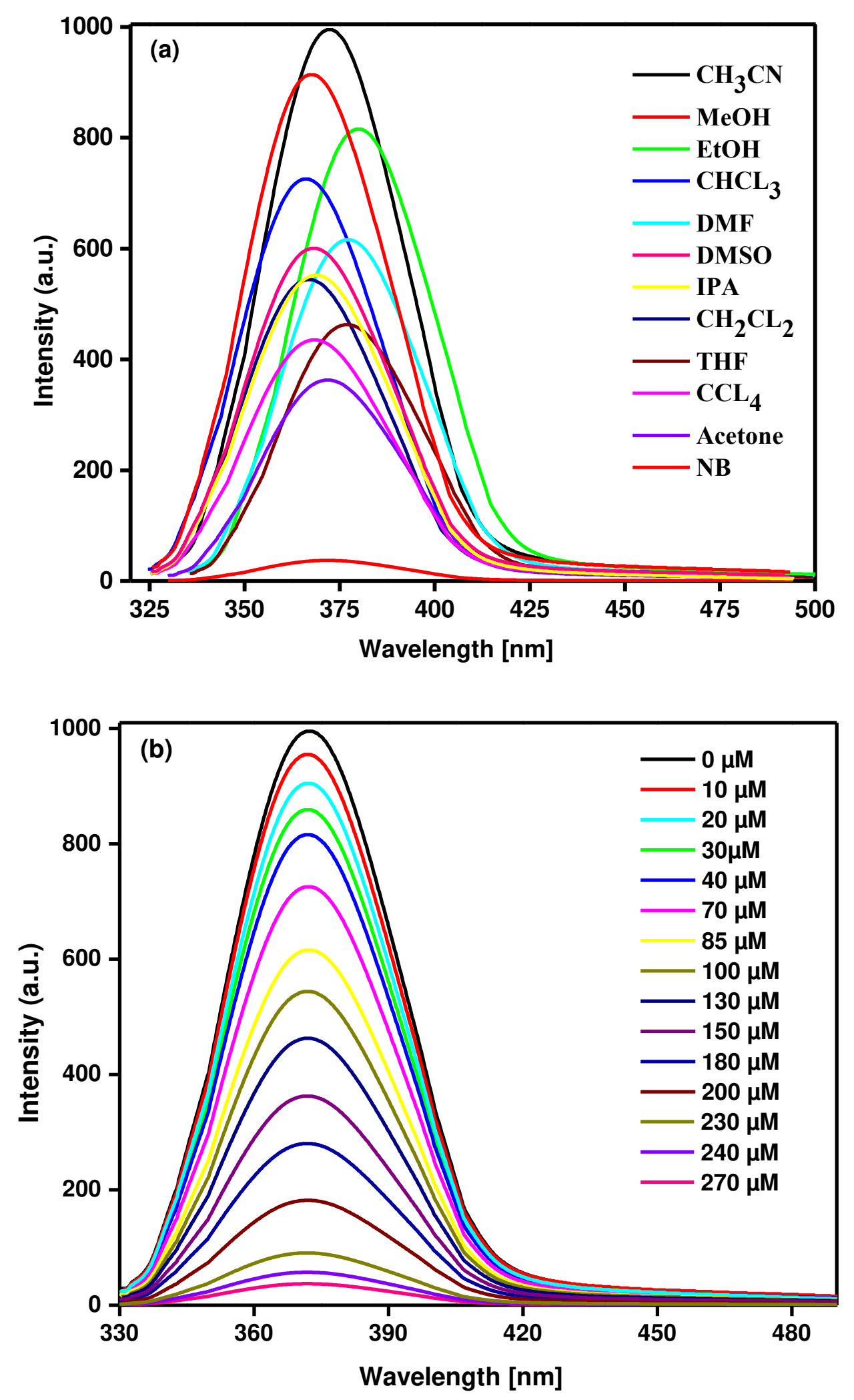


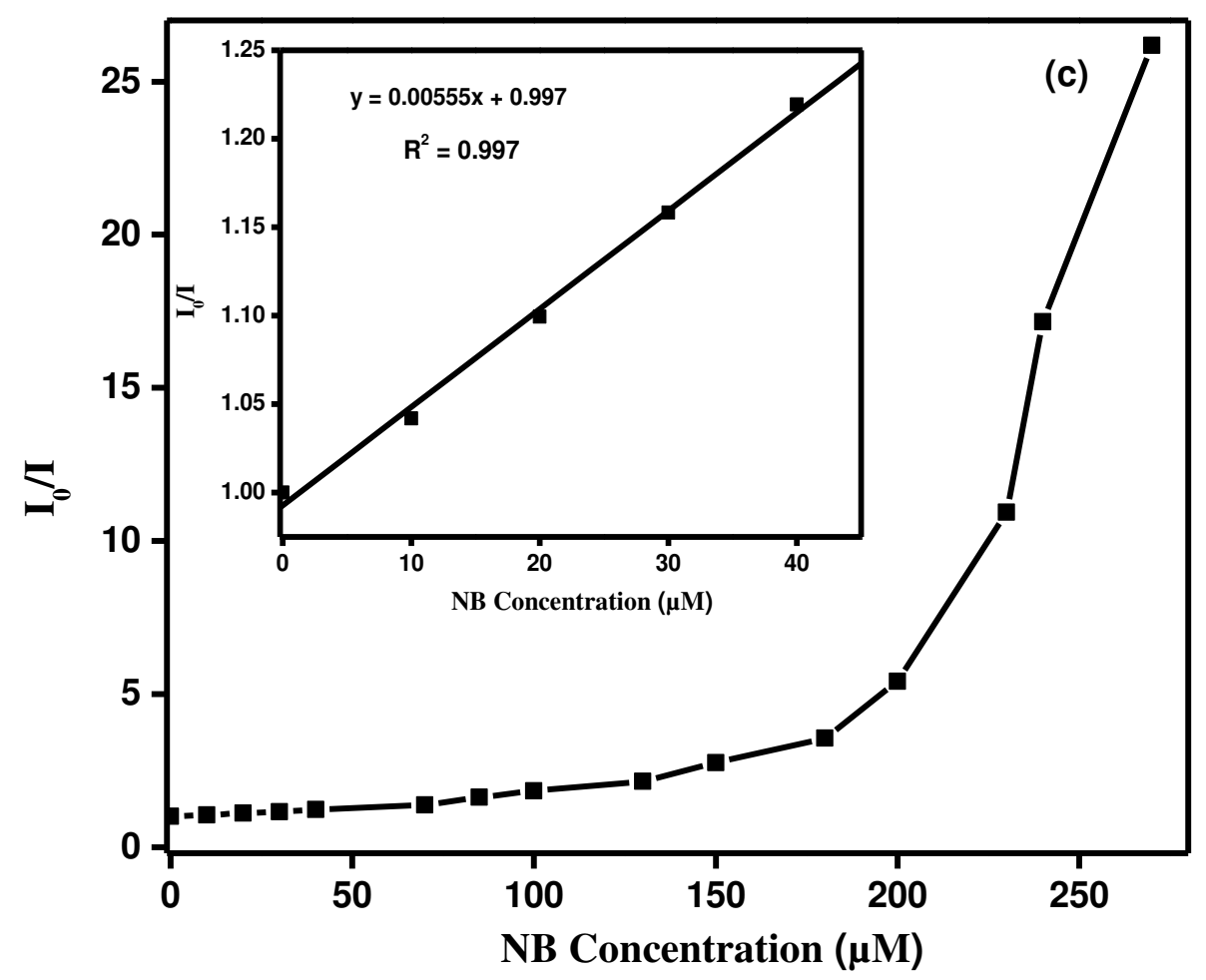

Fig. 6. (a) Luminescence spectra of SCP 1 in different organic solvents, (b) Effect on the emission spectra of SCP 1 suspended in MeCN with the addition of NB concentrations and (c) Stern-Volmer plot of $I_{0} / I$ versus the NB ion concentration. Inset: the fluorescence quenching linearity relationship at low NB concentrations. 

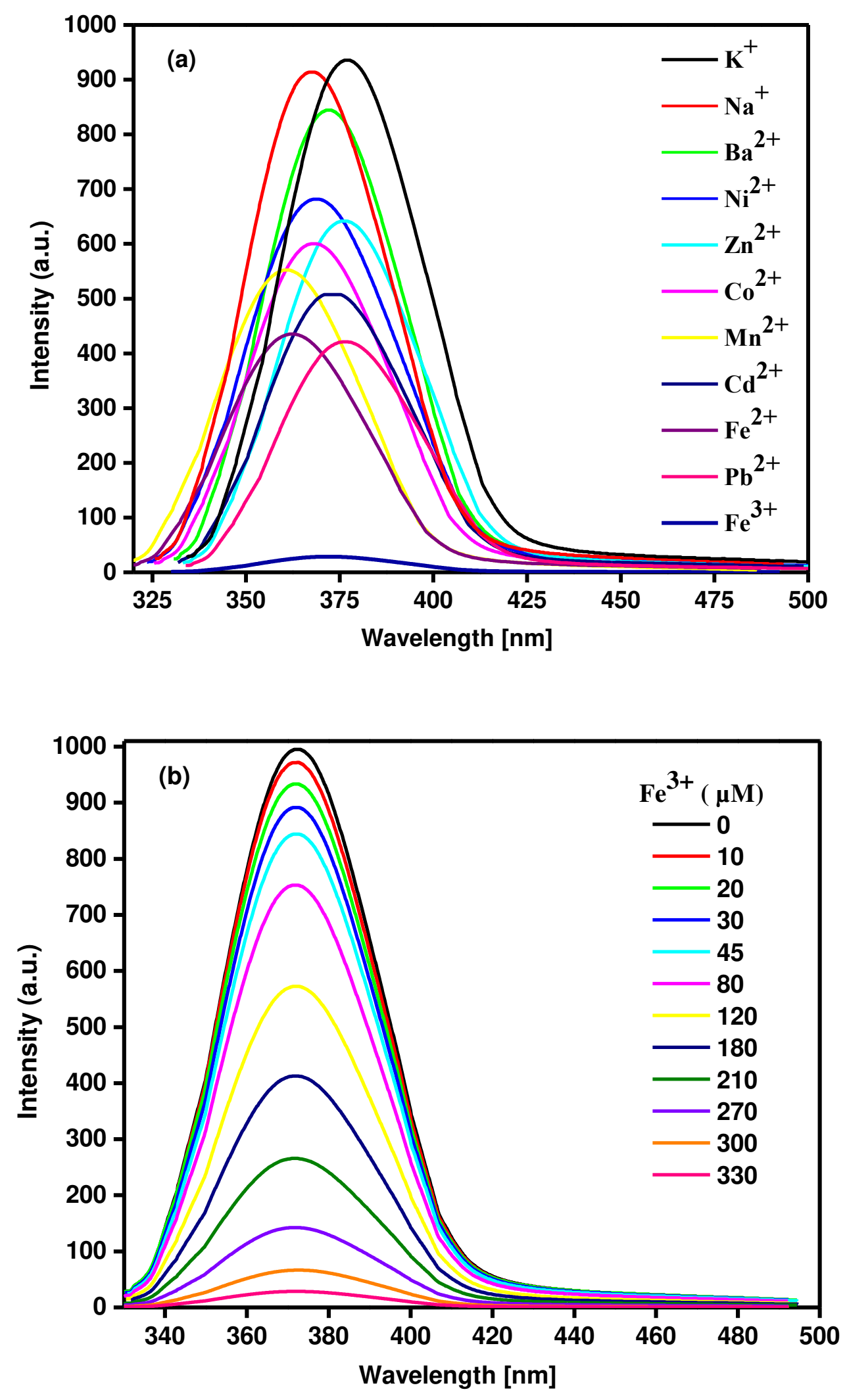


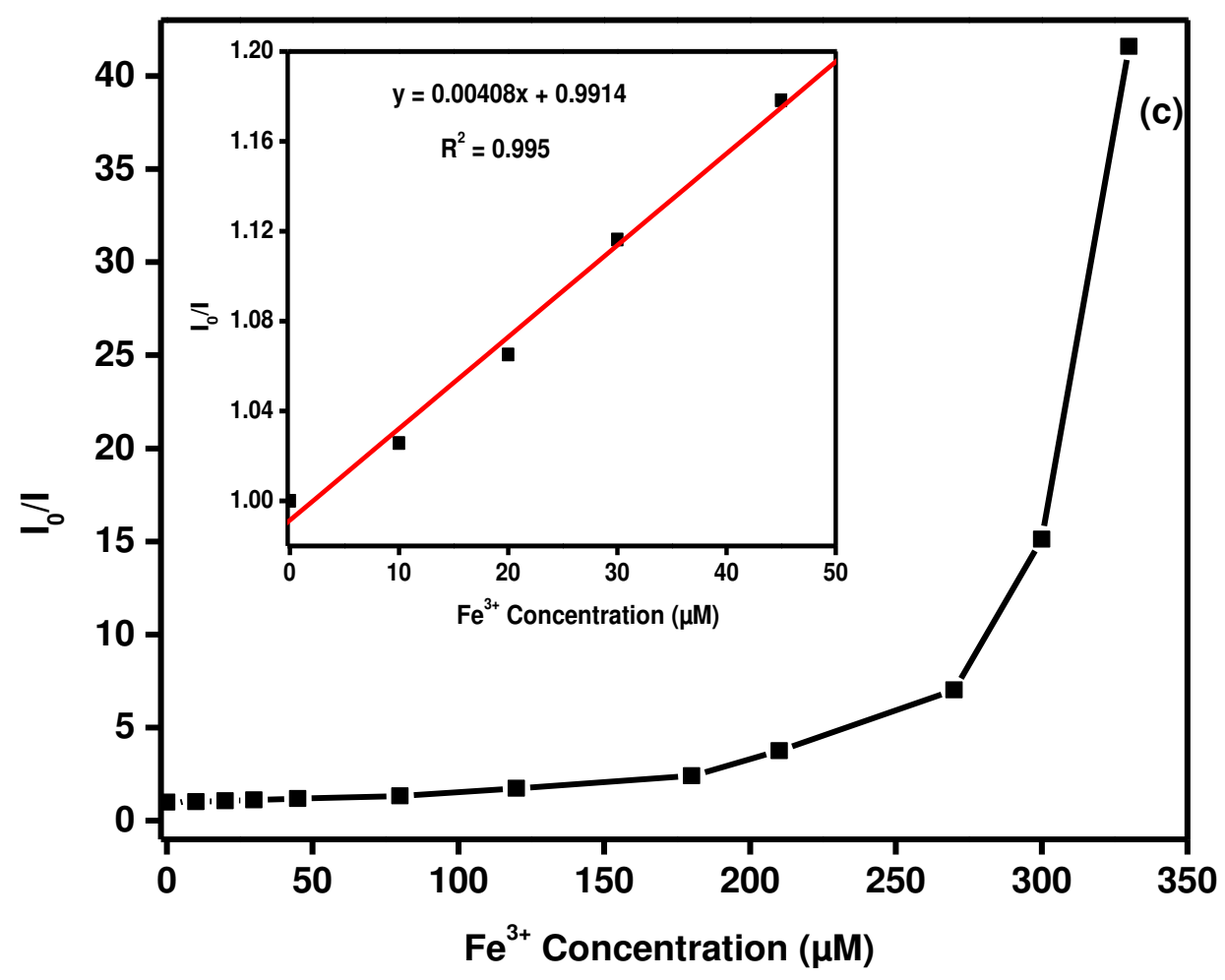

Fig. 7.(a) Luminescence spectra of SCP 1 in aqueous solutions upon gradual addition of different metal ions $\left(10^{-3} \mathrm{M}\right)$; (b) Effect on the emission spectra of SCP 1 suspended in $\mathrm{MeCN}$ with the addition of $\mathrm{Fe}^{3+}$ concentrations and (c) Stern-Volmer plot of $\mathrm{I} 0 / \mathrm{I}$ versus the $\mathrm{Fe}^{3+}$ ion concentration. Inset: the fluorescence quenching linearity relationship at low $\mathrm{Fe}^{3+}$ concentrations. 

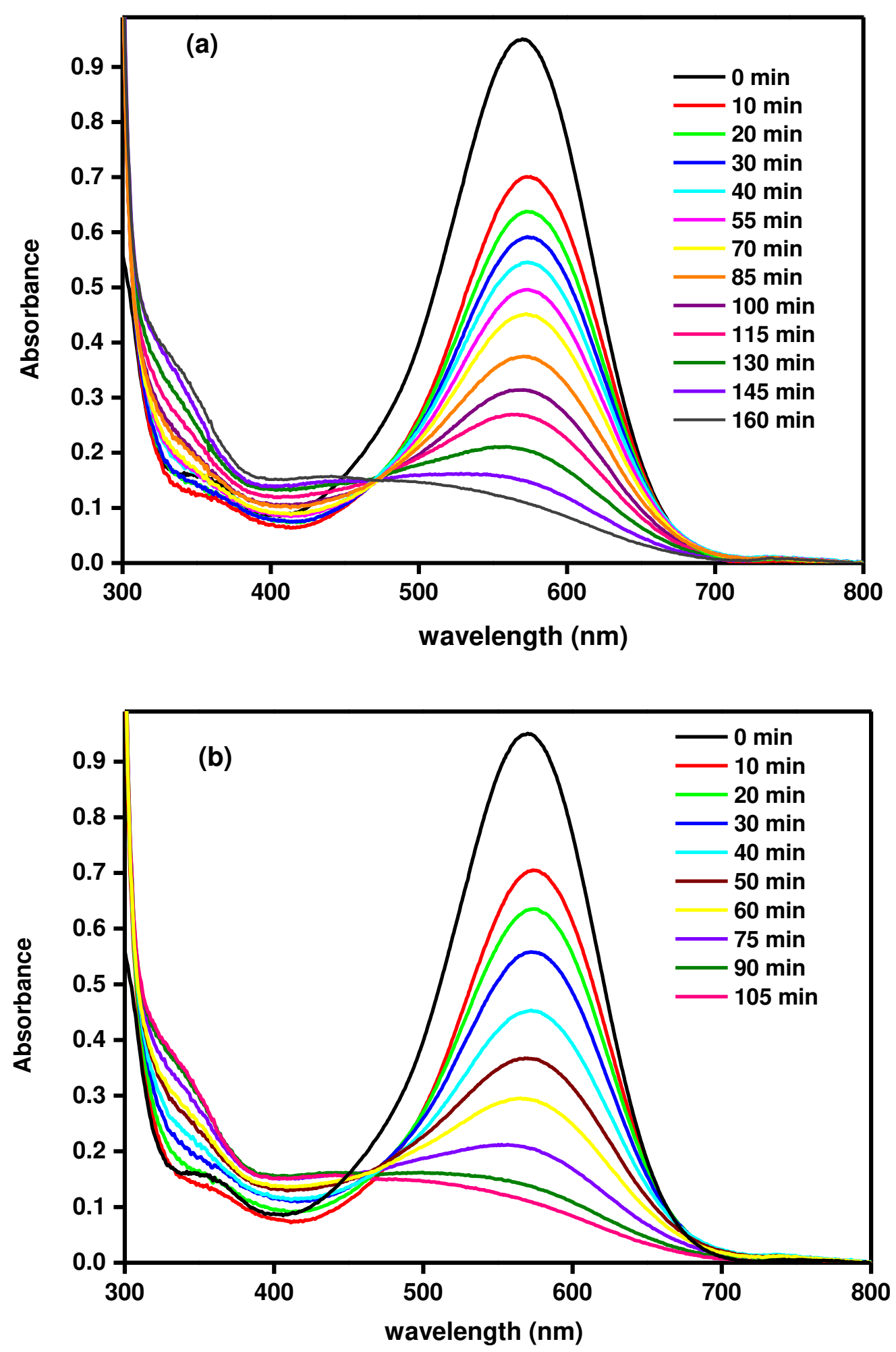

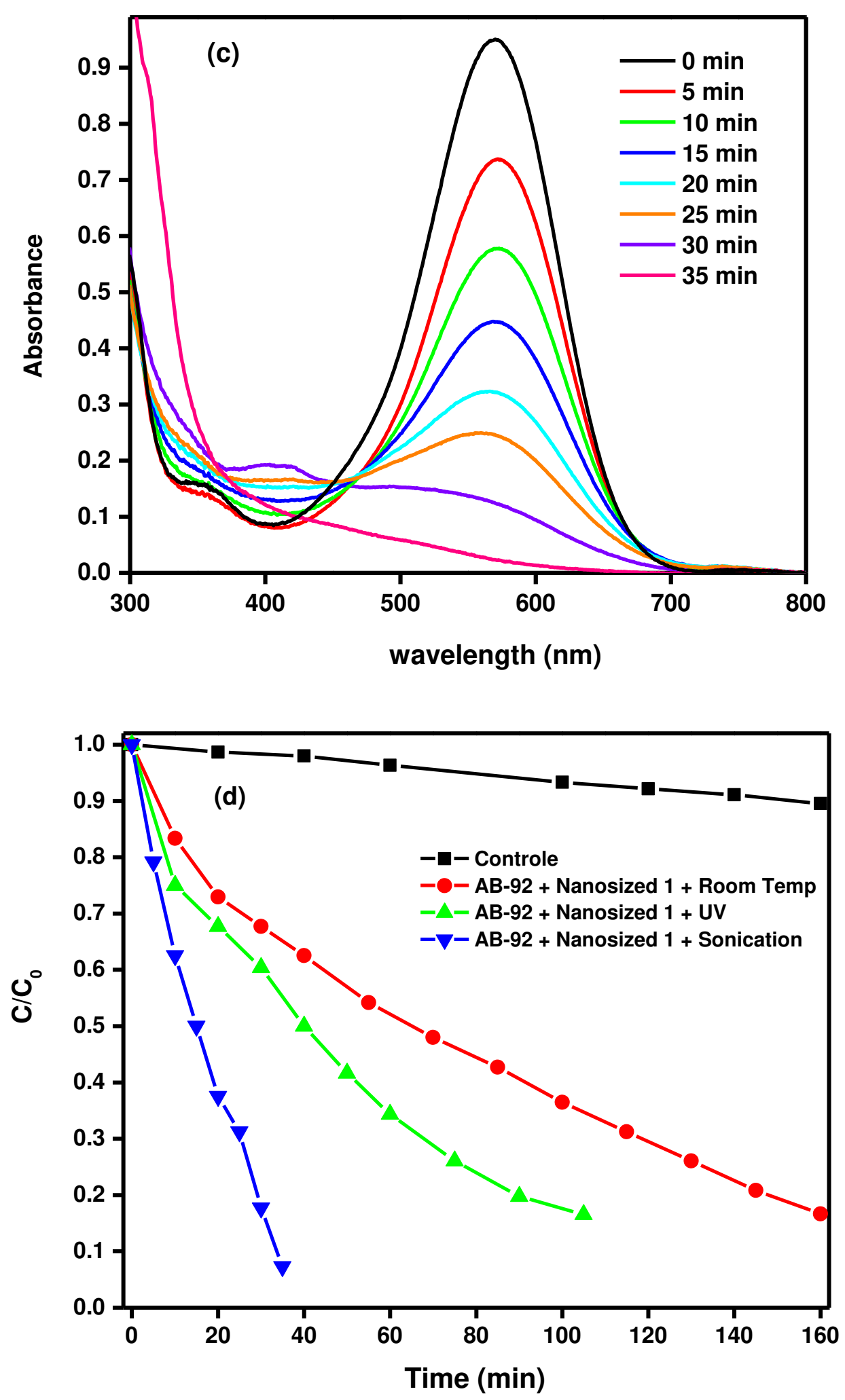

Fig. 8. Temporal evolution of the spectra during the photodegradation of AB-92 dye in the presence of nanosized 2 under (a) Room temperature; (b) under UV-light irradiation; (c) under ultrasonic irradiation and (d) Results of photocatalytic decomposition of AB-92 dye under different irradiation source. 

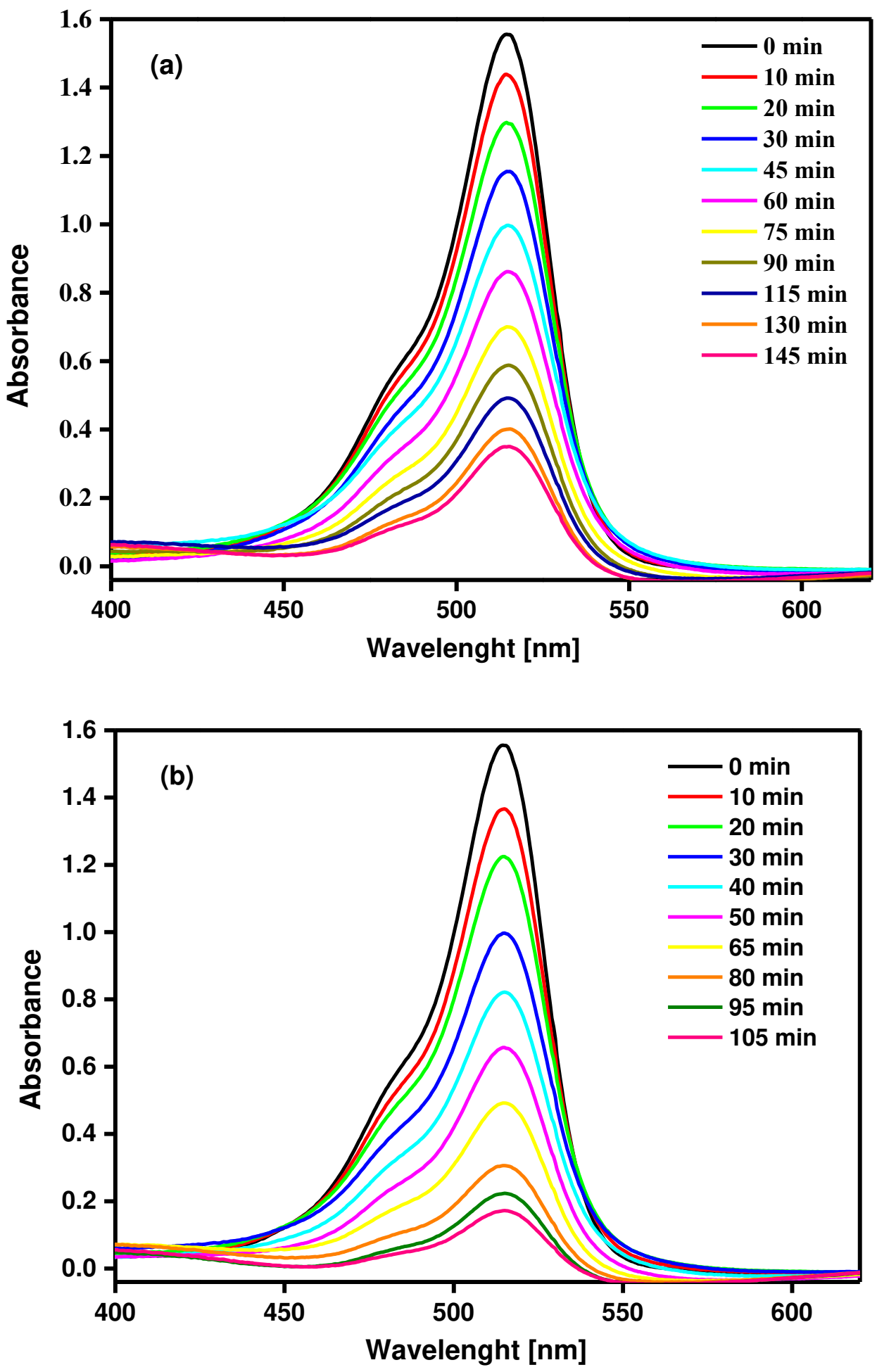

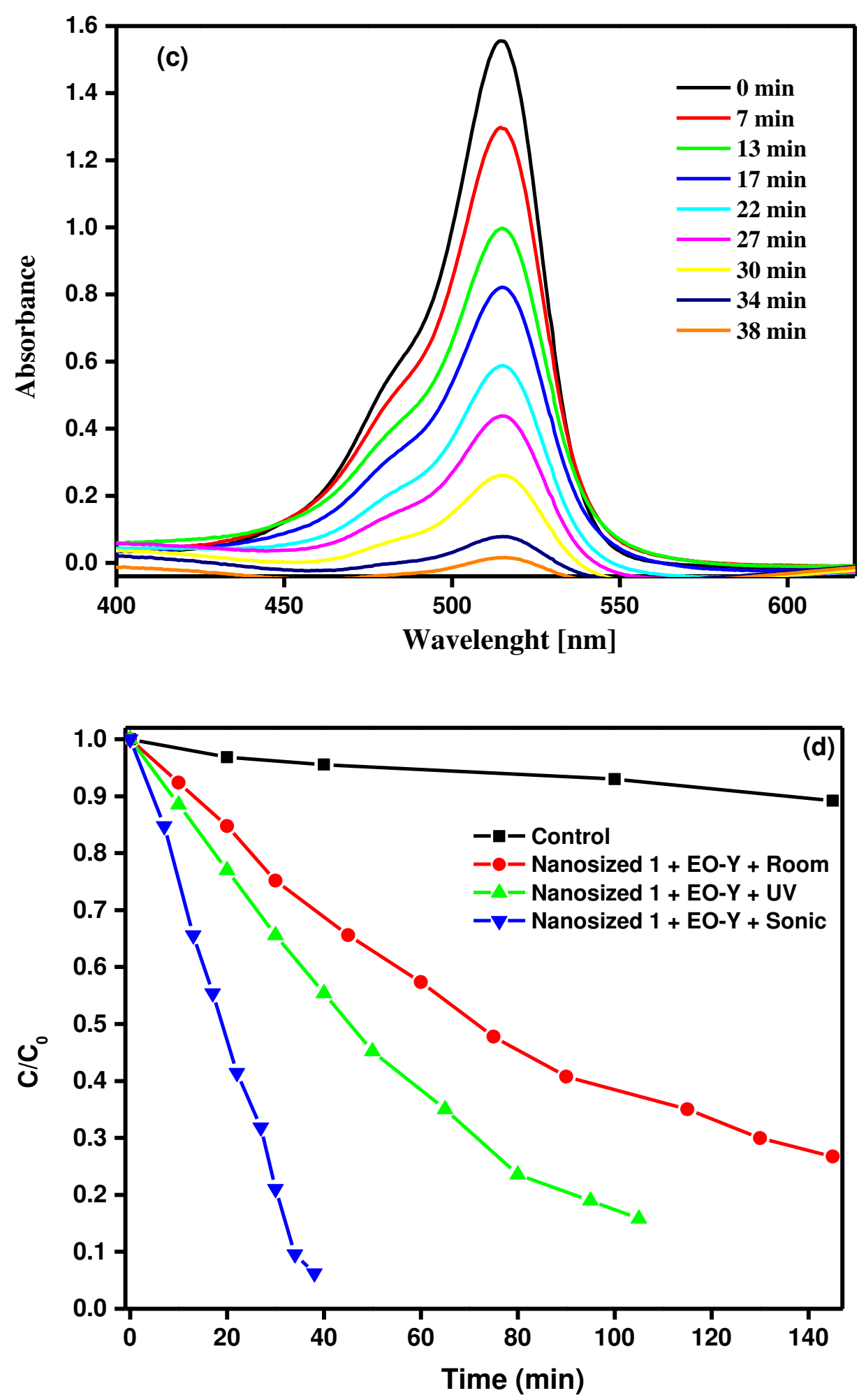

Fig. 9. Temporal evolution of the spectra during the photodegradation of EO-Y dye in the presence of nanosized 2 under (a) Room temperature; (b) under UV-light irradiation; (c) under ultrasonic irradiation and (d) Results of photocatalytic decomposition of EO-Y dye under different irradiation source. 


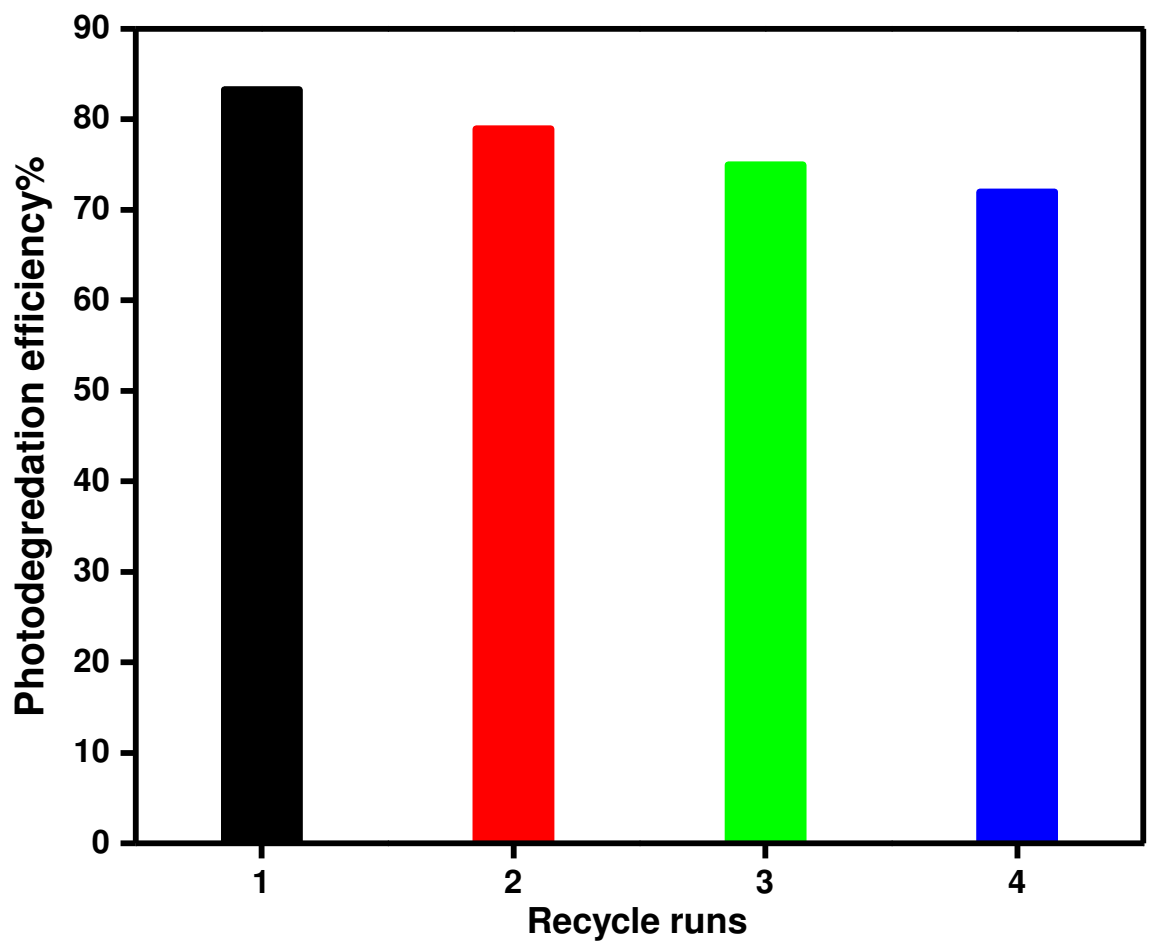

Fig. 10. The recycle runs of the photodegradation of EO-Y dye $\left(2.5 \times 10^{-4} \mathrm{M}\right)$ and $\mathrm{H}_{2} \mathrm{O}_{2}(0.1$ M) in presence of nanosized $\mathbf{2}$ under UV-light irradiation. 


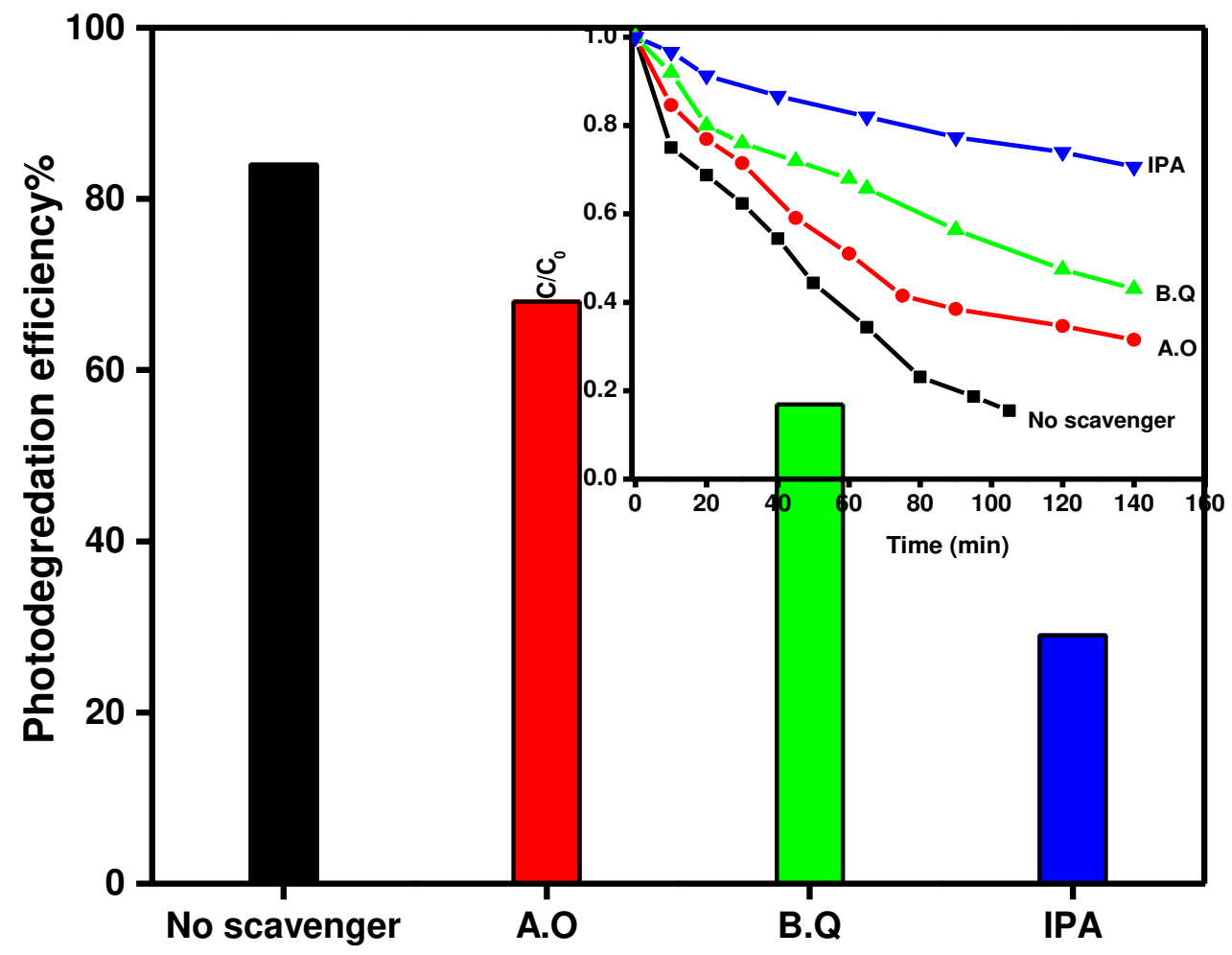

Fig. 11. Effect of different scavengers on the Photo-degradation of EO-Y dye in the presence of nanosized 1 under UV-light irradiation 

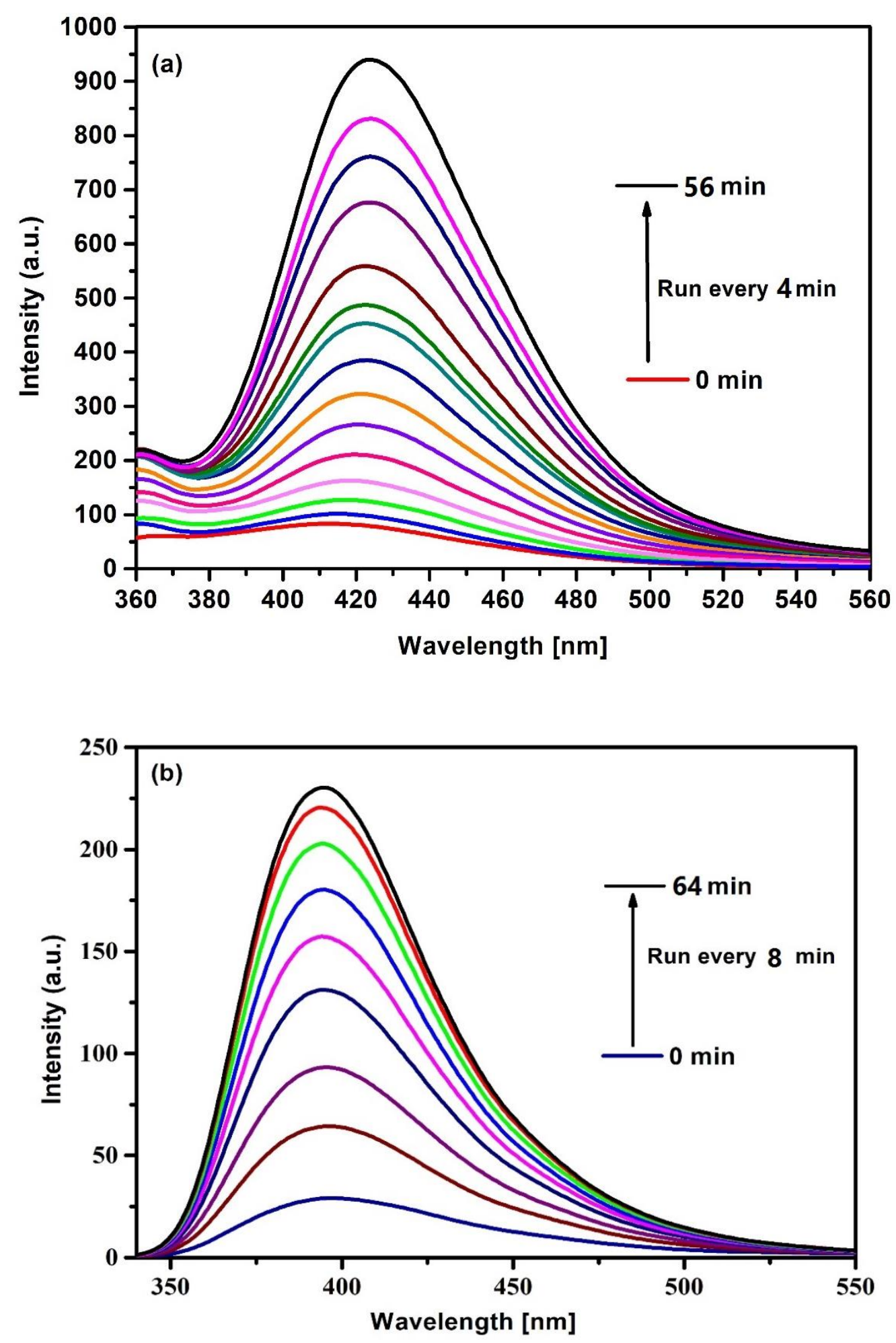

Figure 12 Fluorescence spectra of the solution containing the nanosized 2 catalyst $/ \mathrm{H}_{2} \mathrm{O}_{2}$ system and NaTA $\left(\lambda_{\mathrm{ex}}=340 \mathrm{~nm}\right)(\mathrm{a})$ in absence of AB-92 dye and (b) in presence of AB-92 dye 

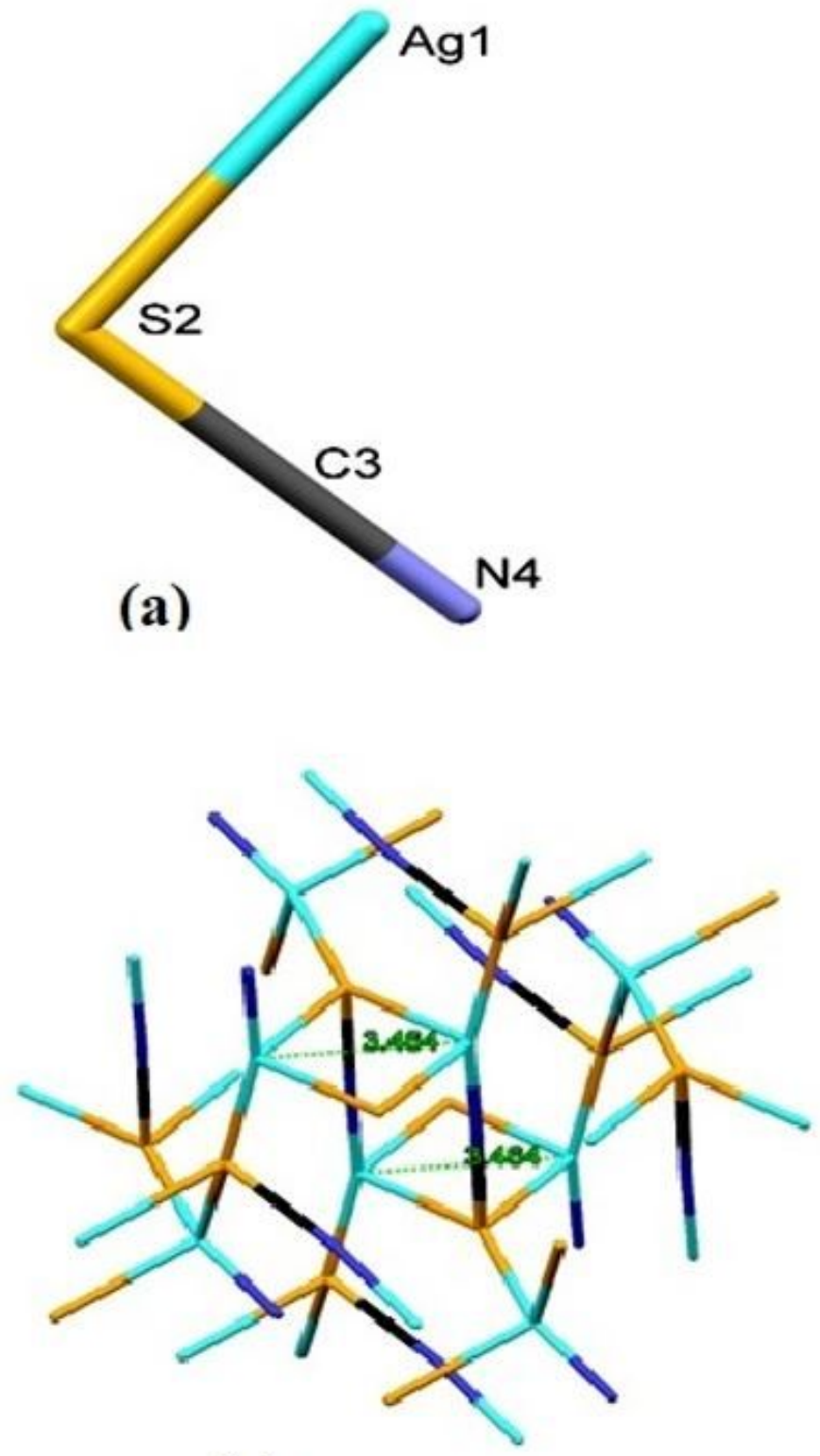

(c)

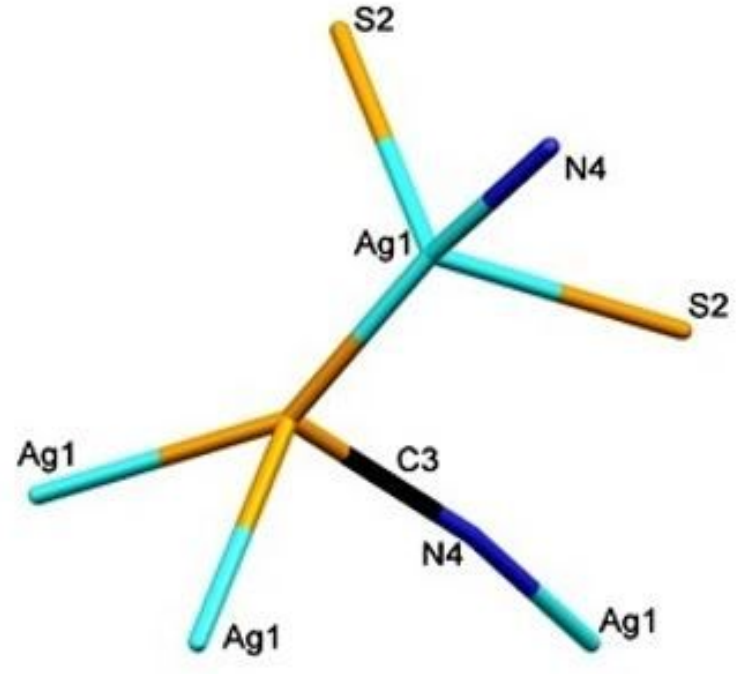

(b)

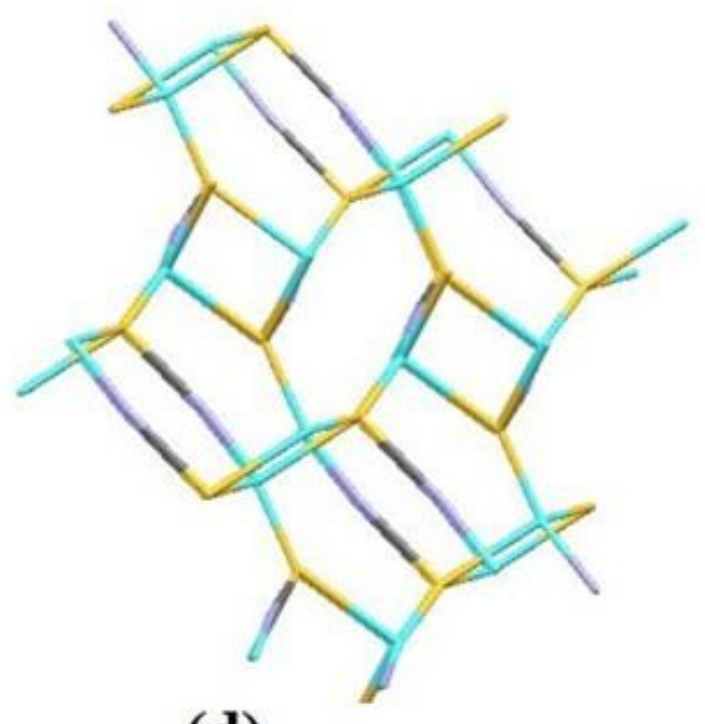

(d)

Figure 1

(a)Asymmetric unit of AgSCN showing atom labeling scheme, (b) view of tetrahedral geometry of AgSCN along a-axis, (c) packing structure showing the complex connections via quadro minicycle (Ag2S2) motifs extending the structure three dimensionally, (d) view of the distorted polygonal rings 


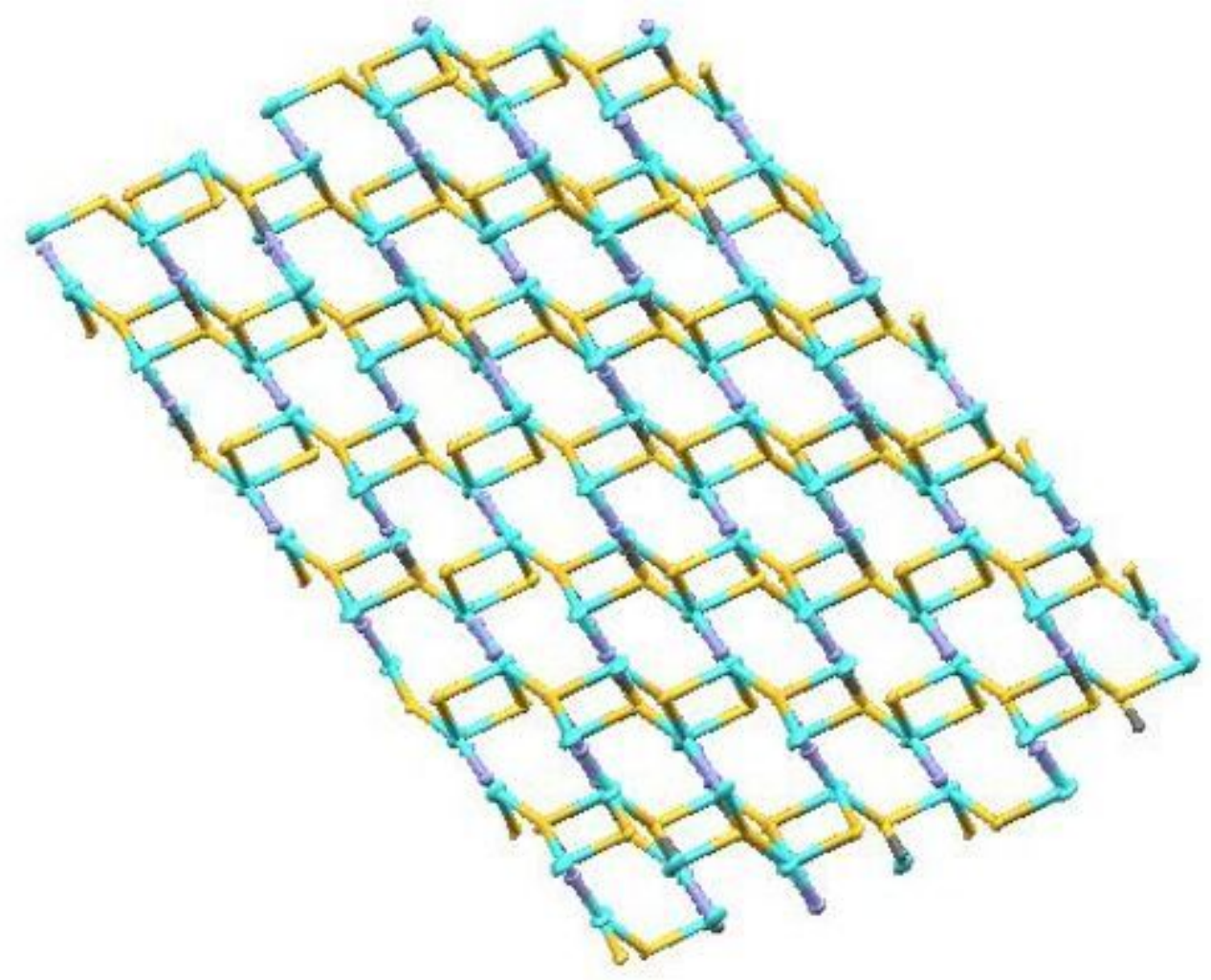

Figure 2

View of the 3D-network structure of SCP1 showing the stair construction along the b-axis 


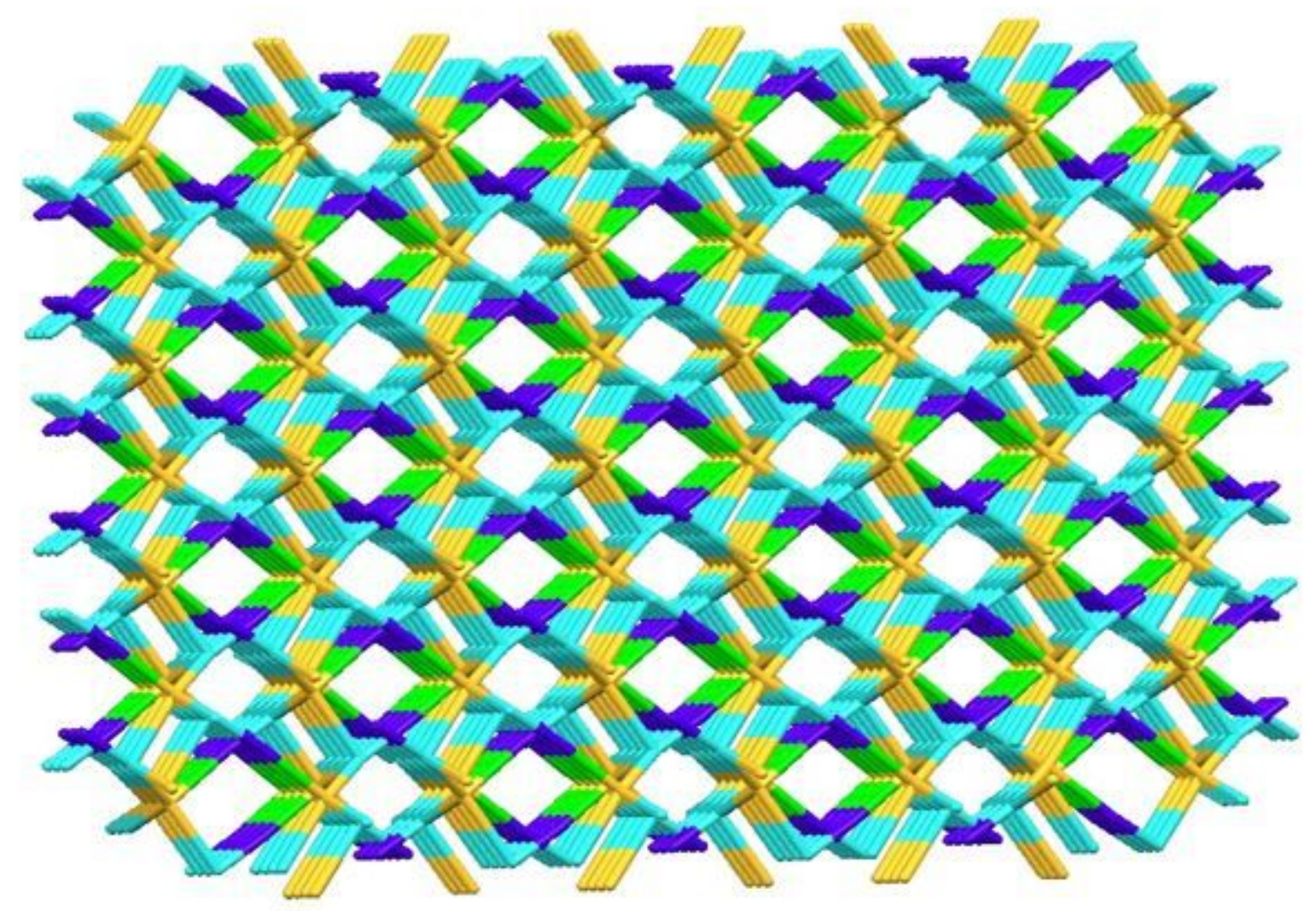

Figure 3

Visualization of the 3D-network structure of SCP1 down the projection of the a -axis showing the pore structure 


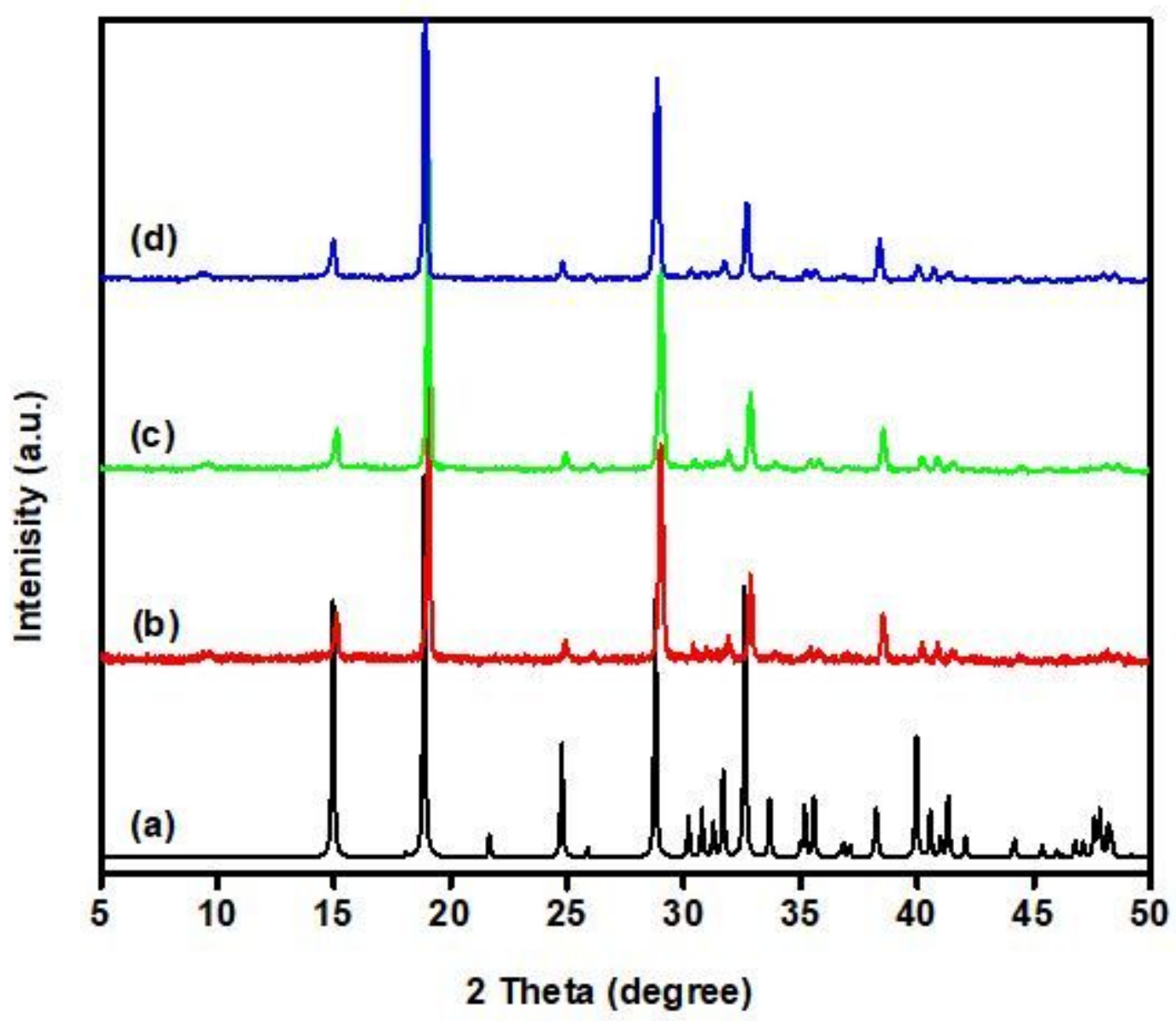

Figure 4

PXRD patterns: (a) simulated pattern based on single crystal data of SCP1; (b) SCP1 as synthesized by self-assembly method; (c) nanosized 2 before degradation process;(d) nanosized 2 after degradation process 


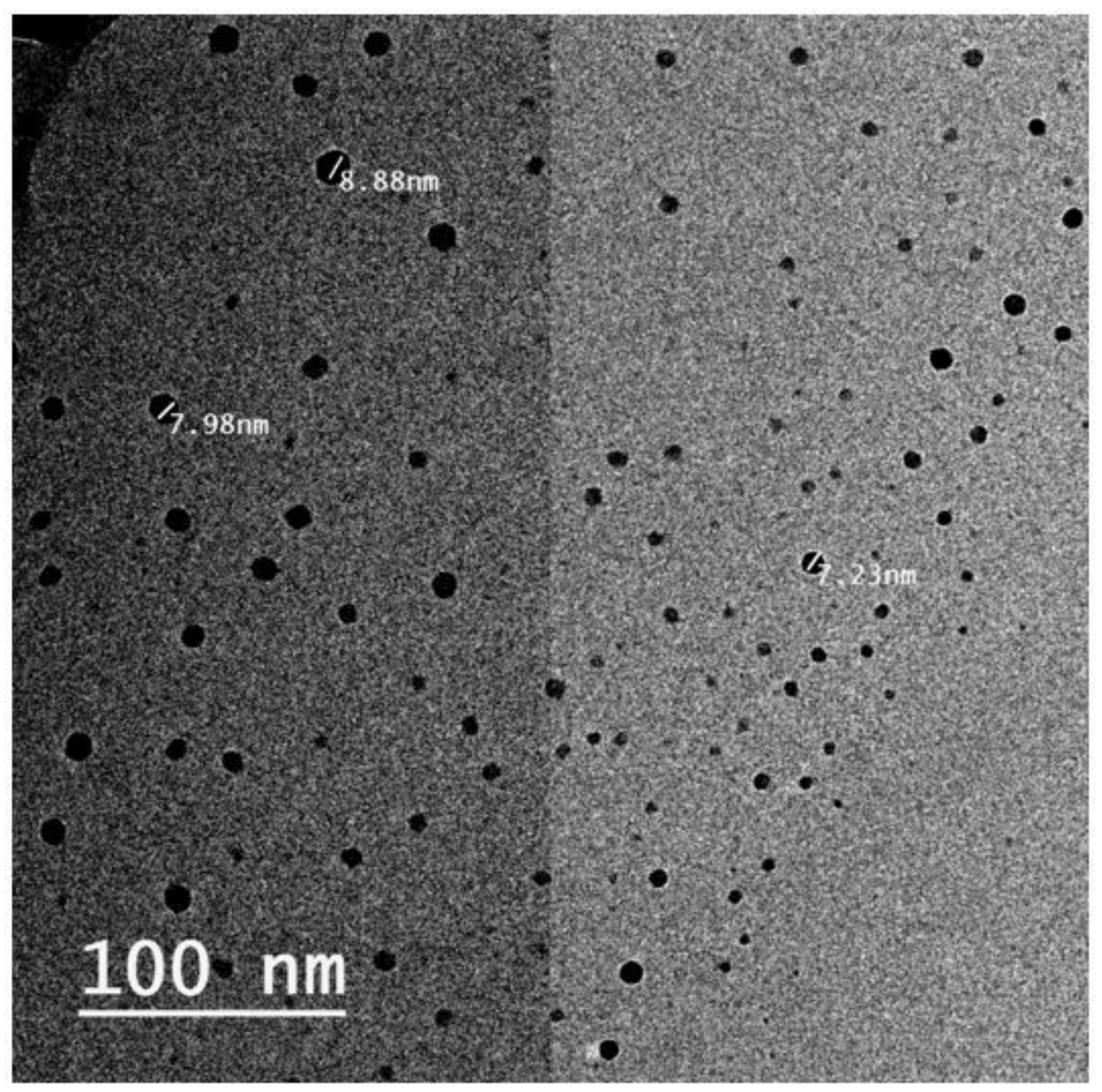

Figure 5

The TEM image of 2 showing the nanosized particles 

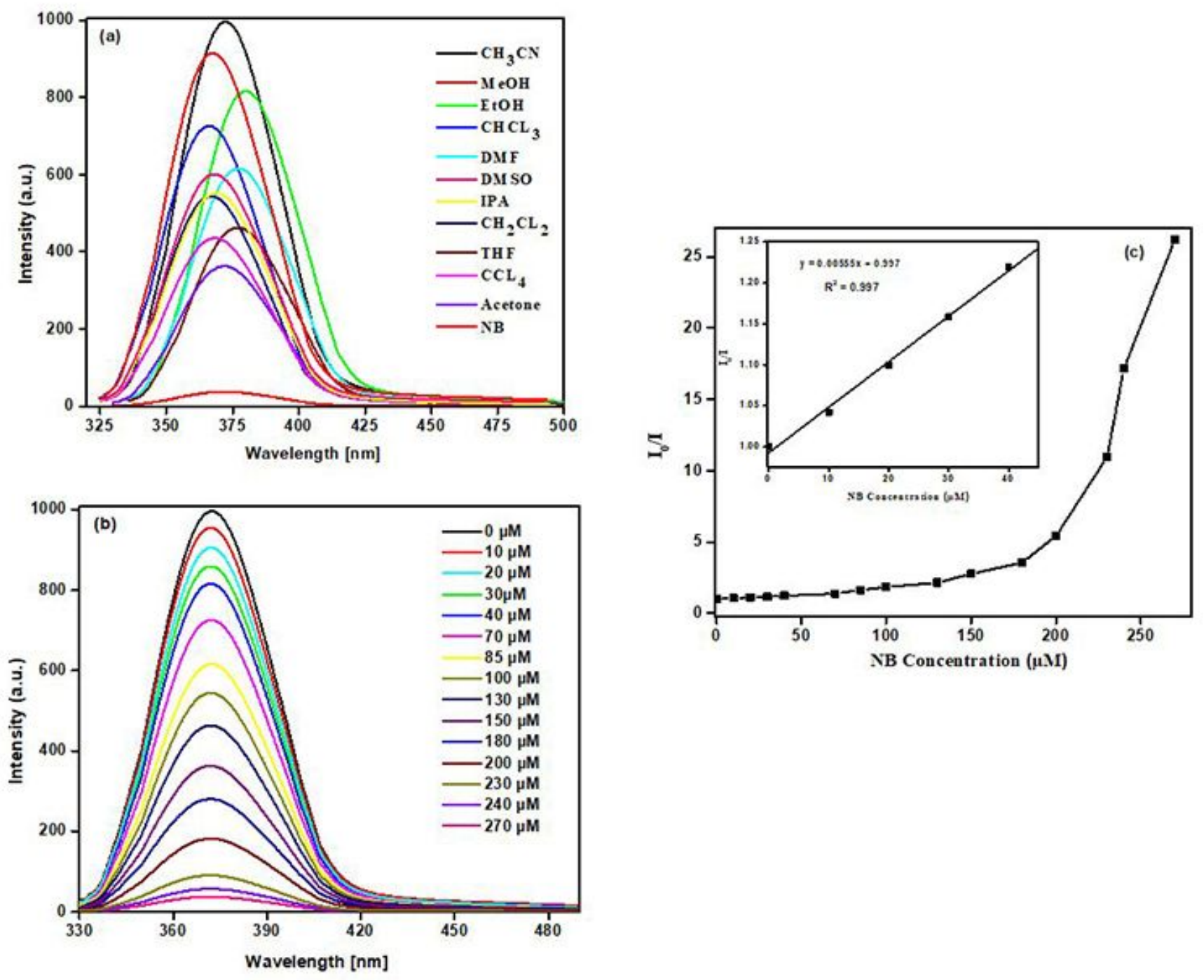

\section{Figure 6}

(a) Luminescence spectra of SCP 1 in different organic solvents, (b) Effect on the emission spectra of SCP 1 suspended in MeCN with the addition of NB concentrations and (c) Stern-Volmer plot of I0/I versus the NB ion concentration. Inset: the fluorescence quenching linearity relationship at low NB concentrations. 

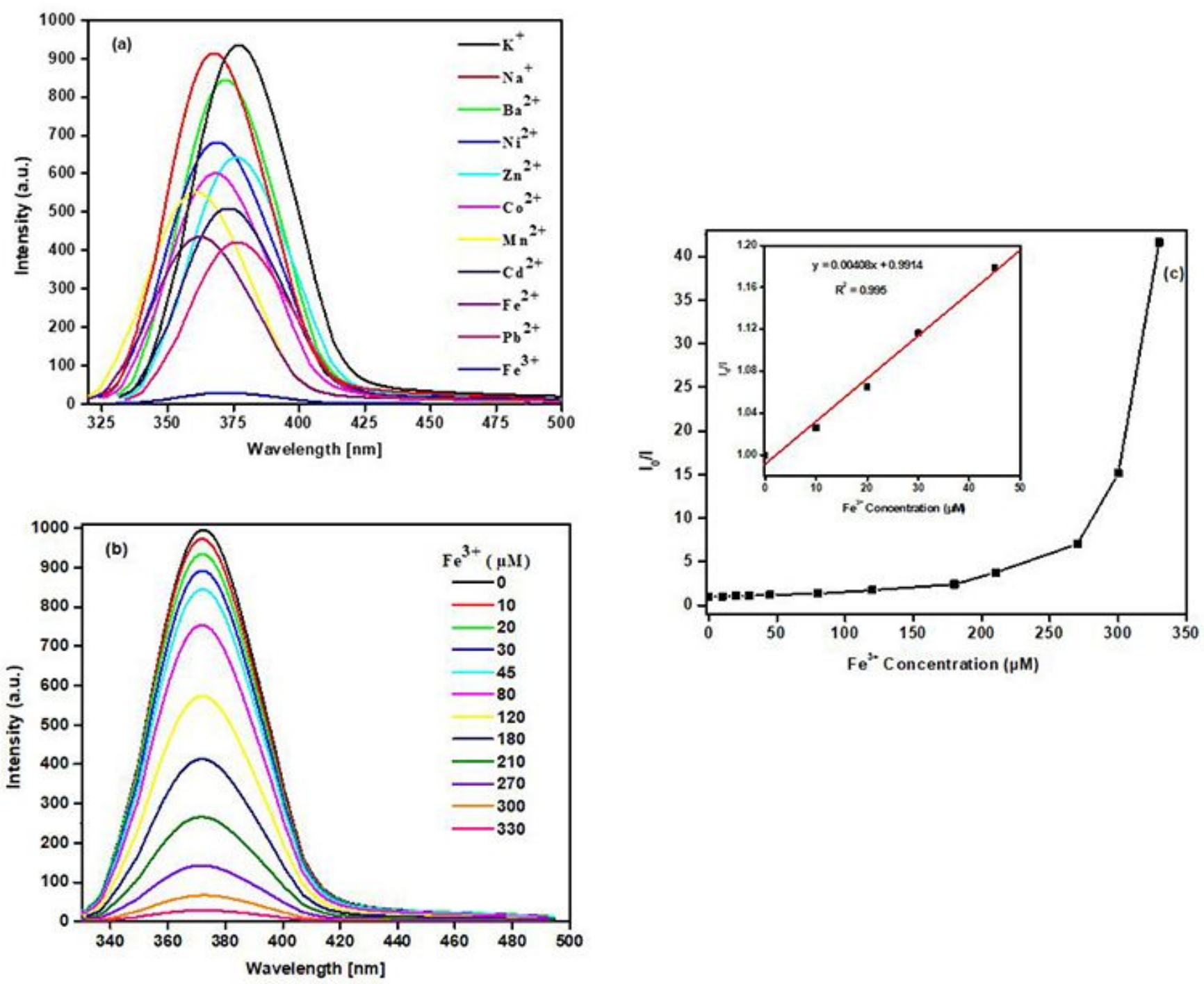

\section{Figure 7}

(a) Luminescence spectra of SCP 1 in aqueous solutions upon gradual addition of different metal ions (10-3M); (b) Effect on the emission spectra of SCP 1 suspended in MeCN with the addition of Fe3+ concentrations and (c) Stern-Volmer plot of I0/I versus the Fe3+ ion concentration. Inset: the fluorescence quenching linearity relationship at low $\mathrm{Fe} 3+$ concentrations. 

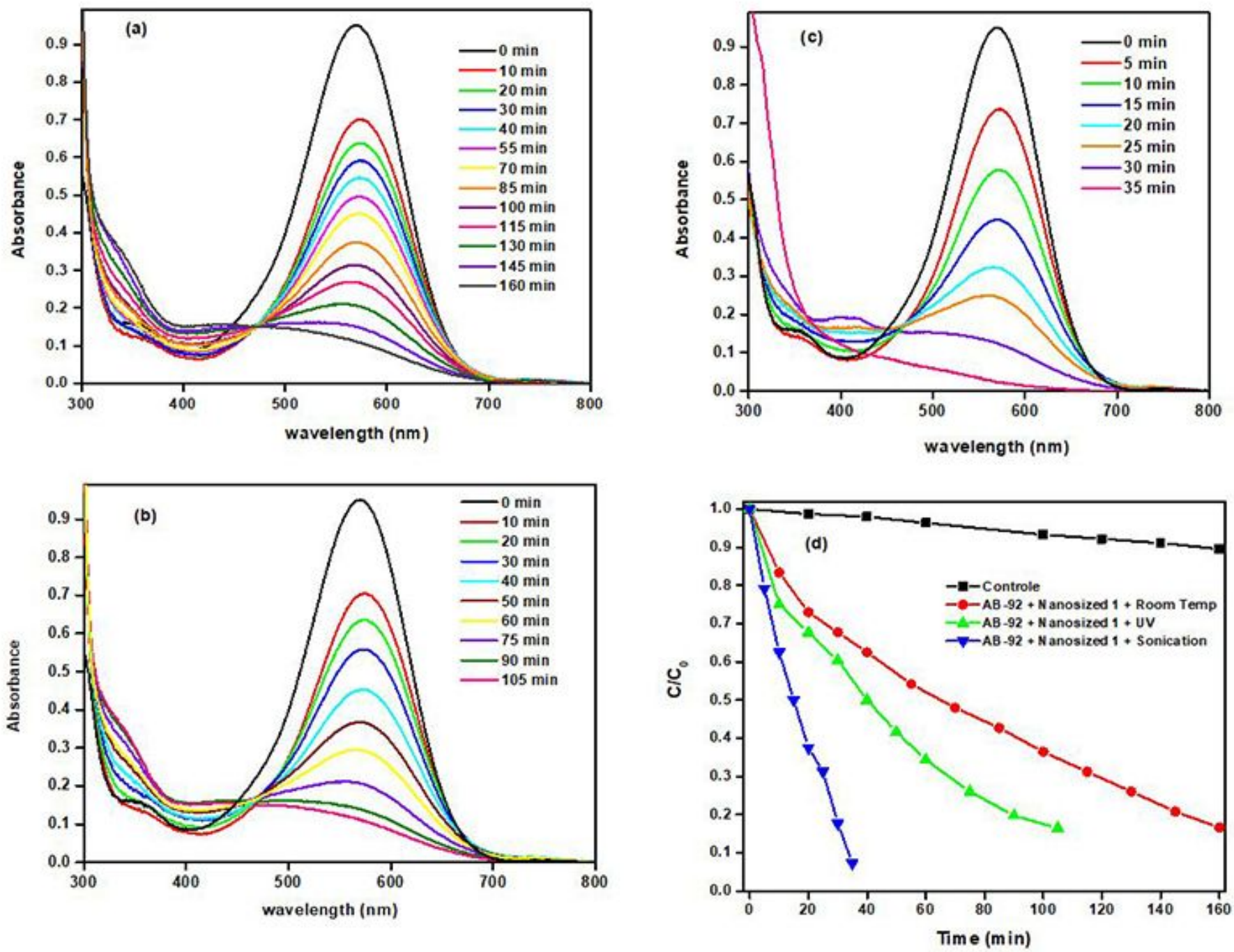

Figure 8

Temporal evolution of the spectra during the photodegradation of AB-92 dye in the presence of nanosized 2 under (a) Room temperature; (b) under UV-light irradiation; (c) under ultrasonic irradiation and (d) Results of photocatalytic decomposition of $A B-92$ dye under different irradiation source. 

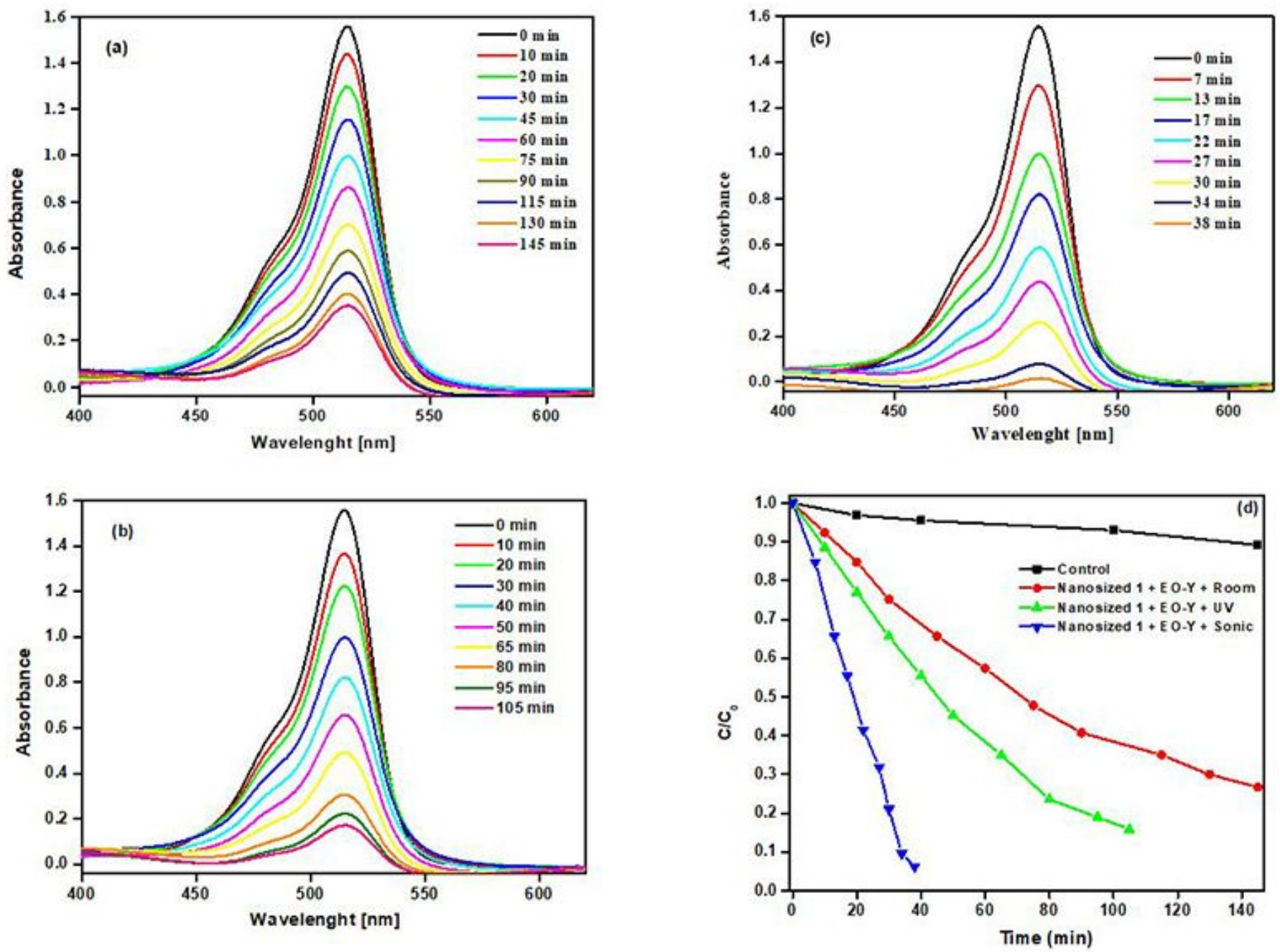

Figure 9

Temporal evolution of the spectra during the photodegradation of EO-Y dye in the presence of nanosized 2 under (a) Room temperature; (b) under UV-light irradiation; (c) under ultrasonic irradiation and (d) Results of photocatalytic decomposition of EO-Y dye under different irradiation source. 


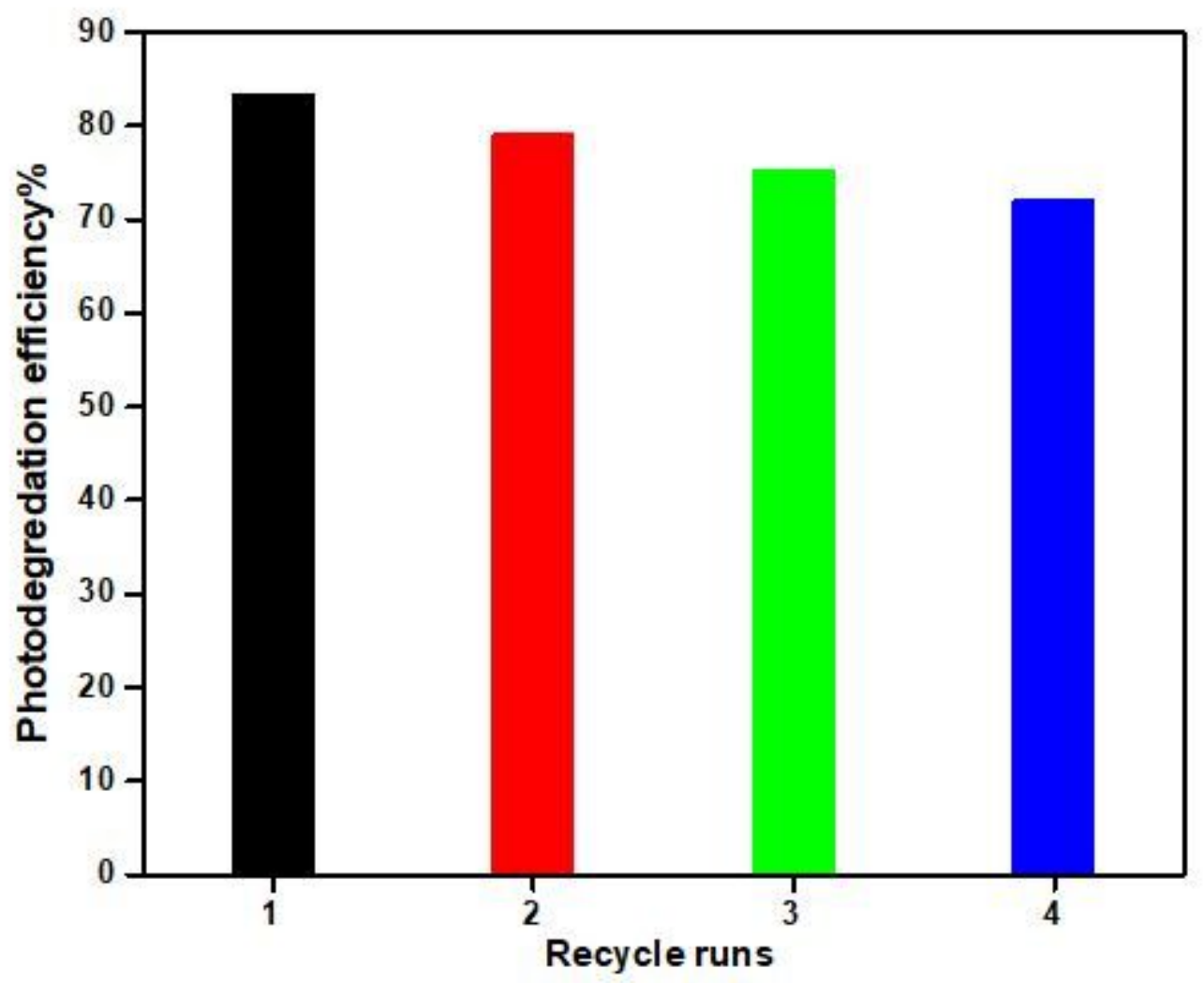

Figure 10

The recycle runs of the photodegradation of EO-Y dye (2.5×10-4 M) and H2O2 (0.1 M) in presence of nanosized 2 under UV-light irradiation. 


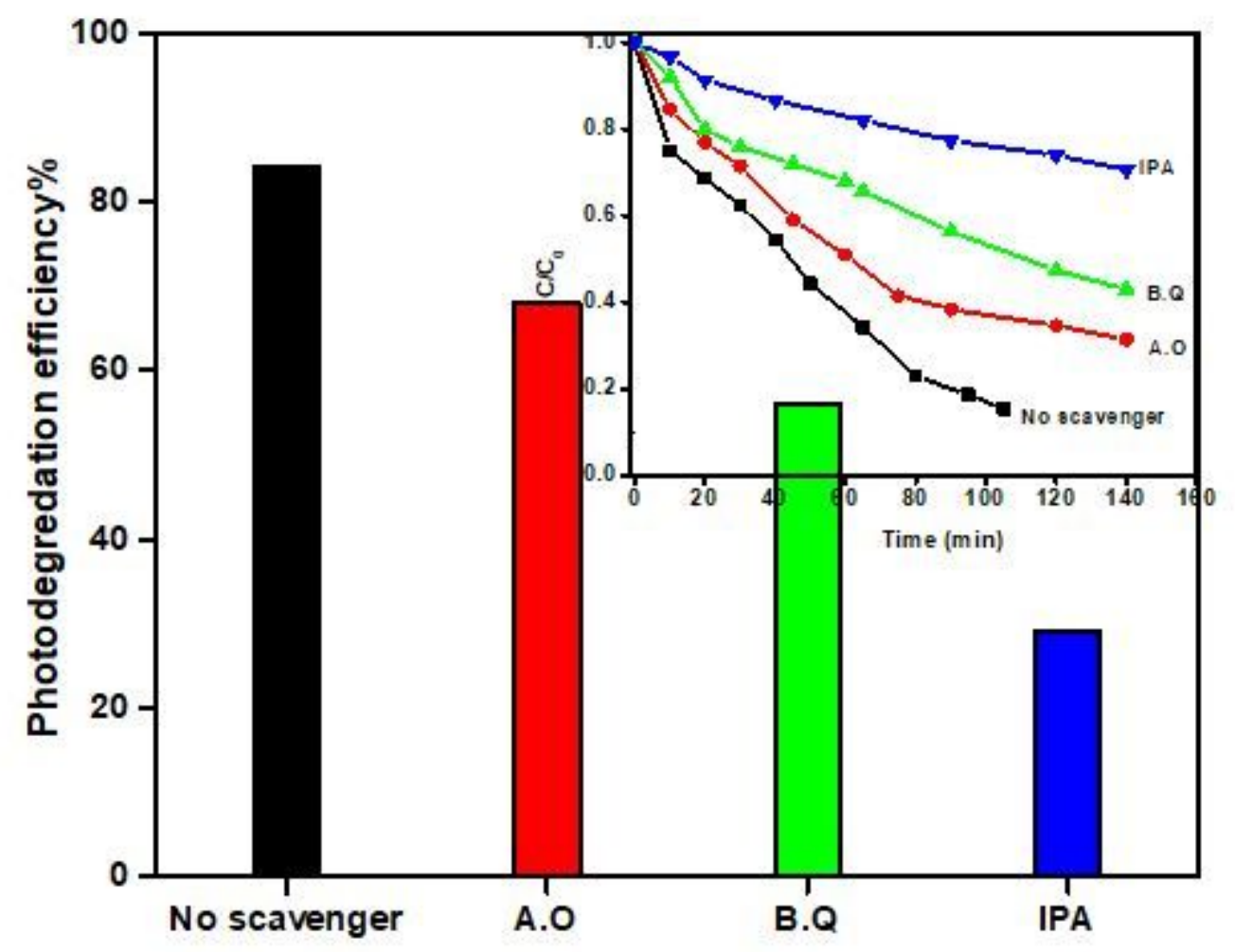

Figure 11

Effect of different scavengers on the Photo-degradation of EO-Y dye in the presence of nanosized 1 under UV-light irradiation 

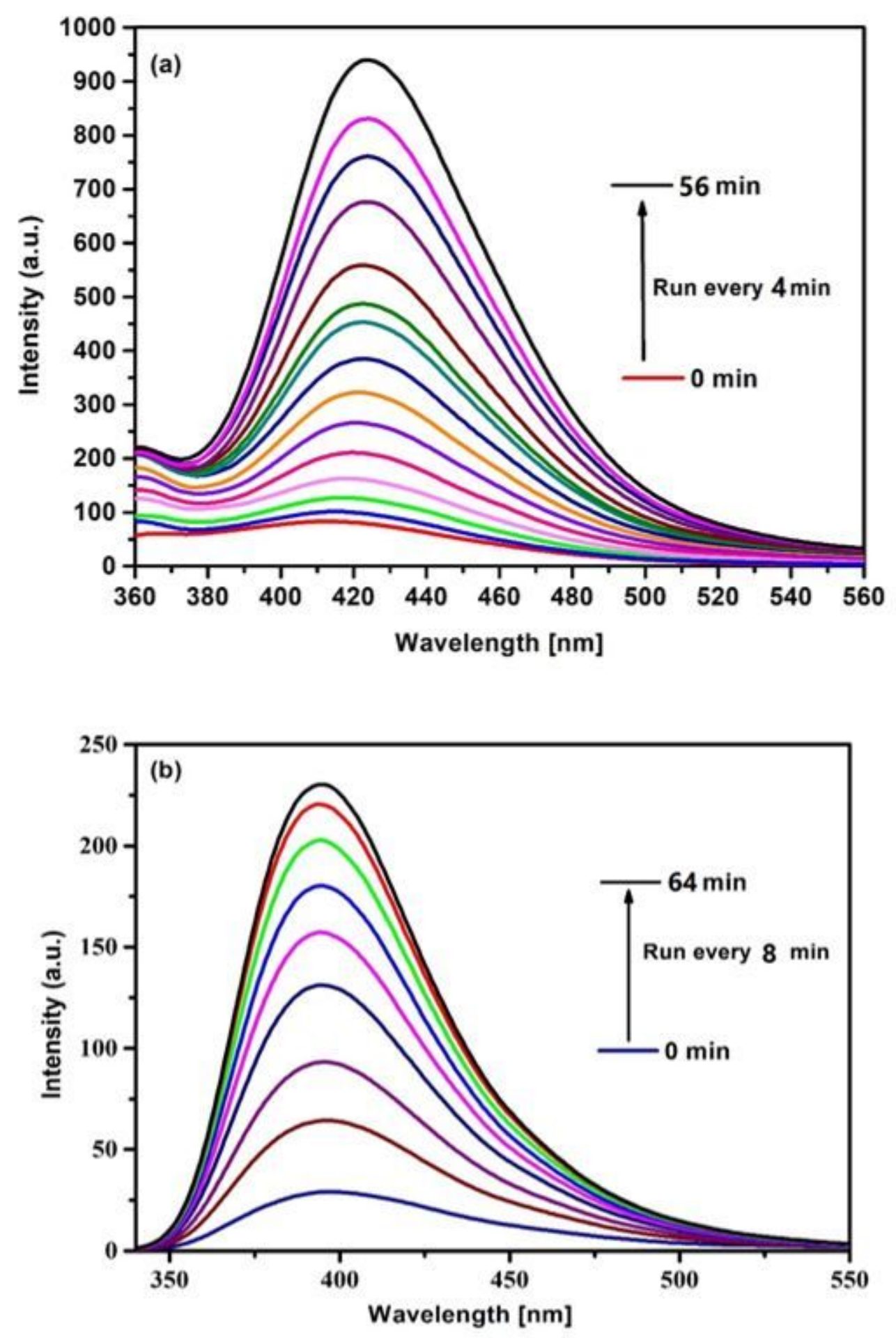

Figure 12

Fluorescence spectra of the solution containing the nanosized 2 catalyst/H2O2 system and $\mathrm{NaTA}(\lambda \mathrm{ex}=$ $340 \mathrm{~nm})(\mathrm{a})$ in absence of $A B-92$ dye and (b) in presence of $A B-92$ dye

\section{Supplementary Files}


This is a list of supplementary files associated with this preprint. Click to download.

- S.B.docx 